\title{
Estimating the average daily rainfall in Thailand using confidence intervals for the common mean of several delta- lognormal distributions
}

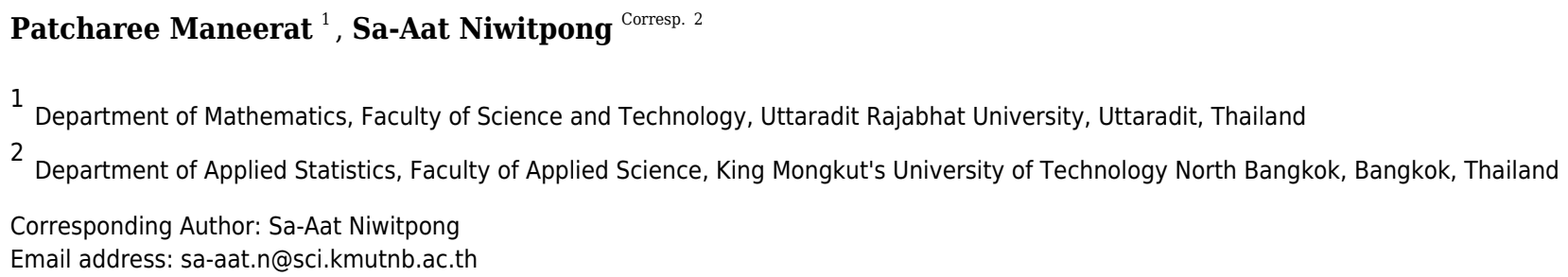

The daily average natural rainfall amounts in the five regions of Thailand can be estimated using the confidence intervals for the common mean of several delta-lognormal distributions based on the fiducial generalized confidence interval $(\mathrm{FGCl})$, large sample (LS), method of variance estimates recovery (MOVER), parametric bootstrap (PB), and highest posterior density intervals based on Jeffreys' rule (HPD-JR) and normal-gammabeta (HPD-NGB) priors. Monte Carlo simulation was conducted to assess the performance in terms of the coverage probability and average length of the proposed methods. The numerical results indicate that MOVER and PB provided better performances than the other methods in a variety of situations, even when the sample case was large. The efficacies of the proposed methods were illustrated by applying them to real rainfall datasets from the five regions of Thailand. 


\title{
Estimating the average daily rainfall in
} Thailand using confidence intervals for the common mean of several delta-lognormal distributions

${ }_{5}$ Patcharee Maneerat ${ }^{1}$ and Sa-Aat Niwitpong ${ }^{2}$

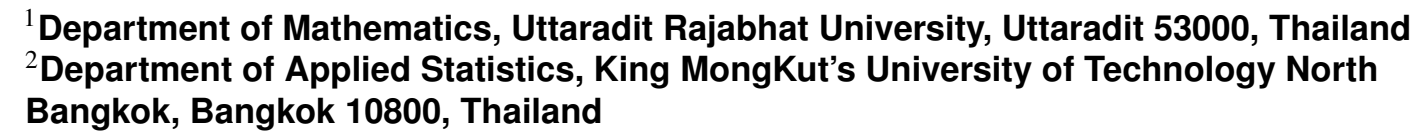

${ }^{1}$ Department of Mathematics, Uttaradit Rajabhat University, Uttaradit 53000, Thailand ${ }^{2}$ Department of Applied Statistics, King MongKut's University of Technology North Bangkok, Bangkok 10800, Thailand

Corresponding author:

Sa-Aat Niwitpong ${ }^{2}$

11 Email address: sa-aat.n@sci.kmutnb.ac.th

\begin{abstract}
The daily average natural rainfall amounts in the five regions of Thailand can be estimated using the confidence intervals for the common mean of several delta-lognormal distributions based on the fiducial generalized confidence interval (FGCI), large sample (LS), method of variance estimates recovery (MOVER), parametric bootstrap (PB), and highest posterior density intervals based on Jeffreys' rule (HPD-JR) and normal-gamma-beta (HPD-NGB) priors. Monte Carlo simulation was conducted to assess the performance in terms of the coverage probability and average length of the proposed methods. The numerical results indicate that MOVER and PB provided better performances than the other methods in a variety of situations, even when the sample case was large. The efficacies of the proposed methods were illustrated by applying them to real rainfall datasets from the five regions of Thailand.
\end{abstract}

\section{INTRODUCTION}

Approximately $82.2 \%$ of Thailand's cultivated land area depends on natural rainfall (Supasod, 2006), thereby indicating its importance for Thai agriculture. However, it is a natural phenomenon with a significant level of uncertainty that can cause natural disasters such as droughts, floods, and landslides. In many countries around the world, extreme rainfall events have been increasing in frequency and duration. On December 5, 2017, Storm Desmond led to heavy rainfall causing flooding in northern England, Southern Scotland, and Ireland (Otto and Oldenborgh, 2017). On July 6-7, 2018, extreme rainfall events such as floods and landslides affected over 5,000 houses, and approximately 1.9 million people in Japan were evacuated from the at-risk area (Oldenborgh, 2018). In mid-September 2019, the amount of rainfall was extreme during Tropical Storm Imelda in Southeast Texas, USA, where over 1,000 people were affected by large-scale flooding and there were 5 deaths (Oldenborgh et al., 2019). Thus, it is necessary to assess how rainfall varies in each region of a country on a daily basis. Due to the climate pattern and meteorological conditions, Thailand is commonly separated into five regions: northern, northeastern, central, eastern, and southern. The rainfall in each region varies widely due to both location and seasonality. Importantly, Thailand's rainfall data include many zeros with probability $\delta>0$ and positive right-skewed data following a lognormal distribution for the remainder of the probability. Thus, applying a delta-lognormal distribution (Aitchison, 1955) is appropriate.

The mean is a measure of the center of a set of observations (Casella and Berger, 2002) that can be used in statistical inference, while functions of the mean such as the ratio or difference between two means can also be used. These parameters have been applied in many research areas, such as medicine, fish stocks, pharmaceutics, and climatology. For example, they have been used for hypothesis testing of the effect of race on the average medical costs between African American and Caucasian patients with type I diabetes (Zhou et al., 1997), to estimate the mean charges for diagnostic tests on patients with 
unstable chronic medical conditions (Zhou and Tu, 2000; Tian, 2005; Tian and Wu, 2007; Li et al., 2013), to estimate the maximum alcohol concentration in men in an alcohol interaction study (Tian and Wu, 2007; Krishnamoorthy and Oral, 2015), to estimate the mean red cod density around New Zealand as an indication of fish abundance (Fletcher, 2008; Wu and Hsieh, 2014), and to estimate the mean of the monthly rainfall totals to compare rainfall in Bloemfontein and Kimberley in South African (Harvey and van der Merwe, 2012).

In practice, the mean has been widely used in many fields, as mentioned before. When independent samples are recorded from several situations, then the common mean is of interest when studying more than one population. Many researchers have investigated methods for constructing confidence interval (CIs) for the common mean of several distributions. For example, Fairweather (1972) proposed a linear combination of Student's t to construct CIs for the common mean of several normal distributions. Jordan and Krishnamoorthy (1996) solved the problem of CIs for the common mean under unknown and unequal variances based on Student's $\mathrm{t}$ and independent $\mathrm{F}$ variables from several normal populations. Krishnamoorthy and Mathew (2003) presented the generalized CI (GCI) and compared it with the CIs constructed by Fairweather (1972), and Jordan and Krishnamoorthy (1996). Later, Lin and Lee (2005) developed a GCI for the common mean of several normal populations. Tian and Wu (2007) provided CIs for the common mean of several lognormal populations using the generalized variable approach, which was shown to be consistently better than the large sample (LS) approach. Lin and Wang (2013) studied the modification of the quadratic method to make inference via hypothesis testing and interval estimation for several lognormal means. Krishnamoorthy and Oral (2015) proposed the method of variance estimates recovery (MOVER) approach for the common mean of lognormal distributions.

As mentioned earlier, many researchers have developed CIs for the common mean of several normal and lognormal distributions. However, there has not yet been an investigation of statistical inference using the common mean of several delta-lognormal distributions. Since the common mean is used to study more than one population, the average precipitation in the five regions in Thailand can be estimated using it as there is an important need to estimate the daily rainfall trends in these regions. Furthermore, the daily rainfall records from the five regions in Thailand satisfy the assumptions for a delta-lognormal distribution. Herein, CIs for the common mean of several delta-lognormal models based on the fiducial GCI (FGCI), LS, MOVER, parametric bootstrap (PB), and highest posterior density (HPD) intervals based on Jeffreys' rule (HPD-JR) and normal-gamma-beta (HPD-NGB) priors are proposed. The outline of this article is as follows. The ideas behind the proposed methods are detailed in Section 2. Numerical computations are reported in Section 3. In Section 4, the daily natural rainfall records of the five regions in Thailand are used to illustrate the efficacy of the methods. Finally, the paper is ended with a discussion and conclusions.

\section{METHODS}

Let $W_{i j}=\left(W_{i 1}, W_{i 2}, \ldots, W_{i n_{i}}\right)$ be random samples drawn from a delta-lognormal distribution, for $i=1,2, \ldots, k$ and $j=1,2, \ldots, n_{i}$. There are three parameters in this distribution: the mean $\mu_{i}$, variance $\sigma_{i}^{2}$ and the probability of obtaining a zero observation $\delta_{i}$. The distribution of $W_{i j}$ is given by

$$
H\left(w_{i j} ; \mu_{i}, \sigma_{i}^{2}, \delta_{i}\right)= \begin{cases}\delta_{i} & ; w_{i j}=0 \\ \delta_{i}+\left(1-\delta_{i}\right) G\left(w_{i j} ; \mu_{i}, \sigma_{i}^{2}\right) & ; w_{i j}>0\end{cases}
$$

where $G\left(w_{i j} ; \mu_{i}, \sigma_{i}^{2}\right)$ is a lognormal distribution function, denoted as $L N\left(\mu_{i}, \sigma_{i}^{2}\right)$ such that $\ln W_{i j} \sim$ $N\left(\mu_{i}, \sigma_{i}^{2}\right)$. The number of zeros has a binomial distribution $n_{i(0)}=\#\left\{j: w_{i j}=0\right\} \sim B\left(n_{i}, \delta_{i}\right)$. The population mean of $W_{i j}$ is given by

$$
\vartheta_{i}=\left(1-\delta_{i}\right) \exp \left(\mu_{i}+\frac{\sigma_{i}^{2}}{2}\right)
$$

The unbiased estimates of $\mu_{i}, \sigma_{i}^{2}$, and $\delta_{i}$ are $\hat{\mu}_{i}=n_{i(1)}^{-1} \sum_{j: w_{i j}>0} \ln W_{i j}, \hat{\sigma}_{i}^{2}=\left(n_{i(1)}-1\right)^{-1} \sum_{j: w_{i j}>0}\left[\ln W_{i j}-\hat{\mu}_{i}\right]^{2}$, and $\hat{\delta}_{i}=n_{i(0)} / n_{i}$, respectively, where $n_{i}=n_{i(0)}+n_{i(1)} ; n_{i(1)}=\#\left\{j: w_{i j}>0\right\}$. Suppose that the deltalognormal mean in (2) for all $k$ populations are the same, then according to Tian and Wu (2007) and Krishnamoorthy and Oral (2015), the common delta-lognormal mean is defined as 


$$
\vartheta=\left(1-\delta_{i}\right) \exp \left(\mu_{i}+\frac{\sigma_{i}^{2}}{2}\right)
$$

${ }_{87}$ For the $i^{t h}$ sample, the estimates of $\vartheta_{i}$ are $\hat{\vartheta}_{i}^{*}=\left(1-\hat{\delta}_{i}\right) \exp \left(\hat{\mu}_{i}+\frac{\hat{\sigma}_{i}^{2}}{2}\right)$ which contains the unbiased estimates

$$
\begin{aligned}
E\left[\hat{\vartheta}_{i}^{*}\right] & =\left[1-E\left(\hat{\delta}_{i}\right)\right] E\left[\exp \left\{\hat{\mu}_{i}+\frac{\hat{\sigma}_{i}^{2}}{2}\right\}\right] \\
& =\left(1-\delta_{i}\right) \exp \left(\mu_{i}+\frac{\sigma_{i}^{2}}{n_{i(1)}}\right)\left(\frac{l_{i}}{l_{i}-\sigma_{i}^{2}}\right)^{l_{i} / 2}
\end{aligned}
$$

89 where $\hat{\delta}_{i} \sim N\left(\delta_{i}, \frac{\delta_{i}\left(1-\delta_{i}\right)}{n_{i}}\right)$ as $n_{i} \rightarrow \infty, E\left[\exp \left(\hat{\mu}_{i}\right)\right]=\exp \left(\mu_{i}+\frac{\sigma_{i}^{2}}{2 n_{i(1)}}\right)$ and $E\left[\exp \left(c_{i} Y_{i}\right)\right]=\left(1-2 c_{i}\right)^{-l / 2}$;

90 $Y_{i}=l_{i} \frac{\hat{\sigma}_{i}^{2}}{\sigma_{i}^{2}} \sim \chi_{l_{i}}^{2}$ and $c_{i}=\frac{\sigma_{i}^{2}}{2 l_{i}}, \hat{\sigma}_{i}^{2}=\left(n_{i(1)}-1\right)^{-1} \sum_{j=1}^{n_{i(1)}}\left[\ln \left(W_{i j}\right)-\hat{\mu}_{i}\right]^{2}$. If $\frac{l_{i}-\sigma_{i}^{2}}{l_{i}}=\exp \left[\frac{-2 \sigma_{i}^{2}}{l_{i}}\left(\frac{1}{2}-\frac{1}{2 n_{i(1)}}\right)\right]$, then 91 we can obtain that

$$
\begin{aligned}
E\left[\hat{\vartheta}_{i}^{*}\right] & =\left(1-\delta_{i}\right) \exp \left(\mu_{i}+\frac{\sigma_{i}^{2}}{2 n_{i(1)}}\right)\left\{\exp \left[\frac{-2 \sigma_{i}^{2}}{l_{i}}\left(\frac{1}{2}-\frac{1}{2 n_{i(1)}}\right)\right]\right\}^{-l_{i} / 2} \\
& =\left(1-\delta_{i}\right) \exp \left(\mu_{i}+\frac{\sigma_{i}^{2}}{2}\right)
\end{aligned}
$$

92 According to Aitchison and Brown (1963), the Aitchison estimate of $\vartheta_{i}$ is expressed as

$$
\hat{\vartheta}_{i}^{(A i t)}= \begin{cases}0 & ; n_{i(1)}=0 \\ w_{i 1} / n_{i} & ; n_{i(1)}=1 \\ \left(1-\hat{\delta}_{i}\right) \exp \left(\hat{\mu}_{i}\right) \psi_{n_{i(1)}}\left(\frac{\hat{\sigma}_{i}^{2}}{2}\right) & ; n_{i(1)}>1\end{cases}
$$

93

where $\psi_{a}(b)$ is a Bessel function defined as

$$
\psi_{a}(b)=1+\frac{(a-1) b}{a}+\frac{(a-1)^{3}}{a^{2} 2 !} \frac{b^{2}}{a+1}+\frac{(a-1)^{5}}{a^{3} 3 !} \frac{b^{3}}{(a+1)(a+3)}+\ldots
$$

${ }_{94}$ To investigate the unbiased estimate $\hat{\vartheta}_{i}^{(A i t)}$, the expected value is

$$
\begin{aligned}
E\left[\hat{\vartheta}_{i}^{(A i t)}\right] & =\sum_{j=1}^{n_{i}} P\left(n_{i(1)}=j\right) E\left[\hat{\vartheta}_{i} \mid n_{i(1)}=j\right] \\
& =0+P\left(n_{i(1)}=1\right) E\left[w_{i 1} / n_{i}\right]+\sum_{j=2}^{n_{i}} P\left(n_{i(1)}=j\right) E\left[\hat{\vartheta}_{i} \mid n_{i(1)}=j\right] \\
& =P\left(n_{i(1)}=1\right) \frac{\exp \left(\mu_{i}+\frac{\sigma_{i}^{2}}{2}\right)}{n_{i}}+\sum_{j=2}^{n_{i}} P\left(n_{i(1)}=j\right) E\left[\frac{n_{i(1)}}{n_{i}} \exp \left(\mu_{i}+\frac{\sigma_{i}^{2}}{2}\right) \mid n_{i(1)}=j\right] \\
& =\sum_{j=0}^{n_{i}} P\left(n_{i(1)}=j\right) E\left[\frac{n_{i(1)}}{n_{i}} \exp \left(\mu_{i}+\frac{\sigma_{i}^{2}}{2}\right) \mid n_{i(1)}=j\right] \\
& =E\left[\frac{n_{i(1)}}{n_{i}} \exp \left(\mu_{i}+\frac{\sigma_{i}^{2}}{2}\right)\right] \\
& =\left(1-\delta_{i}\right) \exp \left(\mu_{i}+\frac{\sigma_{i}^{2}}{2}\right)
\end{aligned}
$$


According to Shimizu and Iwase (1981), the uniformly minimum variance unbiased (UMVU) estimate of $\vartheta_{i}$ is

$$
\hat{\vartheta}_{i}^{(S h i)}=\left\{\begin{array}{cc}
0 & ; n_{i(1)}<1 \\
\frac{n_{i(1)}}{n_{i}} \exp \left(\hat{\mu}_{i}\right)_{0} F_{1}\left(\frac{n_{i(1)}-1}{2} ; \frac{n_{i(1)}-1}{4 n_{i(1)}} S_{i}^{2}\right) & ; n_{i(1)} \geq 1
\end{array}\right.
$$

where $S_{i}^{2}=\sum_{j=1}^{n_{i(1)}}\left[\ln \left(W_{i j}\right)-\hat{\mu}_{i}\right]^{2}$ and ${ }_{0} F_{1}(a ; z)=\sum_{m=0}^{\infty} \frac{z^{m}}{(a)_{m} m !}$

$$
(a)_{m}=\left\{\begin{array}{cc}
1 & ; m=0 \\
a(a+1) \ldots(a+m-1) & ; m \geq 1
\end{array}\right.
$$

95 $111 \quad\left(\mu_{i}+V_{i} \sqrt{\frac{\sigma_{i}^{2}}{n_{i(1)}}}, \frac{\sigma_{i}^{2} U_{i}}{n_{i(1)}-1}\right)$ becomes

$$
\begin{aligned}
E\left[\hat{\vartheta}_{i}^{(S h i)}\right] & =E\left[\frac{n_{i(1)}}{n} \exp \left(\hat{\mu}_{i}\right)_{0} F_{1}\left(\frac{n_{i(1)}-1}{2}, \frac{n_{i(1)}-1}{4 n_{i(1)}} S_{i}^{2}\right)\right] \\
& =\frac{n_{i}\left(1-\delta_{i}\right)}{n_{i}} \exp \left[\mu_{i}+\frac{\sigma_{i}^{2}}{2 n_{i(1)}}\right] \exp \left[\frac{n_{i(1)}-1}{2 n_{i(1)}} \sigma_{i}^{2}\right] \\
& =\left(1-\delta_{i}\right) \exp \left(\mu_{i}+\frac{\sigma_{i}^{2}}{2}\right)
\end{aligned}
$$

where $E\left(n_{i(1)}\right)=n_{i}\left(1-\delta_{i}\right)$. The asymptotic variance of $\hat{\vartheta}_{i}^{(S h i)}$ is given by

$$
\begin{aligned}
\operatorname{Var}\left[\hat{\vartheta}_{i}^{(S h i)}\right]= & \exp \left(2 \mu_{i}+\sigma_{i}^{2}\right)\left[\frac{1}{n_{i}^{2}} \sum_{j=1}^{n_{i}}\left(\begin{array}{c}
n_{i} \\
j
\end{array}\right)\left(1-\delta_{i}\right)^{j} \delta^{n_{i}-j} j^{2} \exp \left(\frac{\sigma_{i}^{2}}{j}\right)\right. \\
& \left.{ }_{0} F_{1}\left(\frac{j-1}{2} ; \frac{(j-1)^{2}}{4 j^{2}} \sigma_{i}^{4}\right)-\left(1-\delta_{i}\right)^{2}\right] \\
= & \frac{\exp \left(2 \mu_{i}+\sigma_{i}^{2}\right)}{n_{i}}\left[\delta_{i}\left(1-\delta_{i}\right)+\frac{1}{2}\left(1-\delta_{i}\right)\left(2 \sigma_{i}^{2}+\sigma_{i}^{4}\right)\right]+O\left(n^{-2}\right)
\end{aligned}
$$

Actually, $\psi_{n_{i(1)}}\left(\frac{\hat{\sigma}_{i}^{2}}{2}\right)={ }_{0} F_{1}\left(\frac{n_{i(1)}-1}{2} ; \frac{n_{i(1)}-1}{4 n_{i(1)}} S_{i}^{2}\right)$ such that $\hat{\vartheta}_{i}^{(S h i)}$ and $\hat{\vartheta}_{i}^{(A i t)}$ are the unbiased estimates of $\vartheta_{i}$ under different ideas, although their variances are the same i.e. $\operatorname{Var}\left[\hat{\vartheta}_{i}^{(S h i)}\right]=\operatorname{Var}\left[\hat{\vartheta}_{i}^{(A i t)}\right]$. Using $\hat{\mu}_{i}, \hat{\sigma}_{i}^{2}$, and $\hat{\delta}_{i}$ from the samples, the estimated delta-lognormal mean $\hat{\vartheta}_{i}^{(A i t)}$ and variance of $\hat{\vartheta}_{i}^{(A i t)}$ are obtained. The following methods are the detailed construction of the CIs for the common delta-lognormal mean.

\section{Fiducial Generalized Confidence Interval}

Fiducial inference was introduced by Fisher (1930). Fisher's fiducial argument was used to develop a generalized fiducial recipe that could be extended to the application of fiducial ideas (Hannig, 2009). The concept of the fiducial interval has been advanced by the idea of the generalized pivotal quantity (GPQ) such that it is directly used to apply for generalized inference. Later, Hannig et al. (2006) argued that a subclass of GPQs, the fiducial GPQ (FGPQ), provides a framework that shows the connection between a distribution and a parameter. Recall that $\hat{\mu}_{i} \sim N\left(\mu_{i}, \sigma_{i}^{2} / n_{i(1)}\right)$ and $\left(n_{i(1)}-1\right) \hat{\sigma}_{i}^{2} / \sigma_{i}^{2} \sim \chi_{n_{i(1)}-1}^{2}$ are the independent random variables. The structure functions of $\hat{\mu}_{i}$ and $\hat{\sigma}_{i}^{2}$ are

$$
\hat{\mu}_{i}=\mu_{i}+V_{i} \sqrt{\frac{\sigma_{i}^{2}}{n_{i(1)}}} \quad \text { and } \quad \hat{\sigma}_{i}^{2}=\frac{\sigma_{i}^{2} U_{i}}{n_{i(1)}-1}
$$

which are the function of $V_{i}$ and $U_{i}$, respectively, where $V_{i} \sim N(0,1)$ and $U_{i} \sim \chi_{n_{i(1)}-1}^{2}$. Given the observed values, the estimates $\hat{\mu}_{i}$ and $\hat{\sigma}_{i}^{2}$ can be obtained, and the unique solution of $\left(\hat{\mu}_{i}, \hat{\sigma}_{i}^{2}\right)=$ 


$$
\mu_{i}=\hat{\mu}_{i}-V_{i} \frac{\hat{\sigma}_{i}}{\sqrt{n_{i(1)}}} \sqrt{\frac{n_{i(1)}-1}{U_{i}}}, \quad \sigma_{i}^{2}=\frac{\left(n_{i(1)}-1\right) \hat{\sigma}_{i}^{2}}{U_{i}}
$$

The respective FGPQs of $\mu_{i}$ and $\sigma_{i}^{2}$ are

$$
\begin{aligned}
G_{\mu_{i}} & =\hat{\mu}_{i}-V_{i}^{*} \frac{\hat{\sigma}_{i}}{\sqrt{n_{i(1)}}} \sqrt{\frac{n_{i(1)}-1}{U_{i}^{*}}} \\
G_{\sigma_{i}^{2}} & =\frac{\left(n_{i(1)}-1\right) \hat{\sigma}_{i}^{2}}{U_{i}^{*}}
\end{aligned}
$$

where $V_{i}^{*}$ and $U_{i}^{*}$ are independent copies of $V_{i}$ and $U_{i}$, respectively. Hasan and Krishnamoorthy (2018) developed the FGPQ of $\delta_{i}$ using a beta distribution as $G_{\delta_{i}^{\prime}} \sim \operatorname{Beta}\left(\alpha_{i}, \beta_{i}\right) ; \alpha_{i}=n_{i(1)}+0.5$ and $\beta_{i}=n_{i(0)}+0.5$. The FGPQ of $\vartheta$ based on $k$ individual samples is

$$
G_{\vartheta}=\frac{\sum_{i=1}^{k} G_{w_{i}} G_{\vartheta_{i}}}{\sum_{i=1}^{k} G_{w_{i}}}
$$

where $G_{\vartheta_{i}}=G_{\delta_{i}^{\prime}} \exp \left(G_{\mu_{i}}+G_{\sigma_{i}^{2}} / 2\right), G_{w_{i}}=1 / G_{\operatorname{Var}}\left[\hat{\vartheta}_{i}^{(A i t)}\right]$, and

$G_{\operatorname{Var}\left[\hat{\vartheta}_{i}^{(A i t)}\right]}=\exp \left(2 G_{\mu_{i}}+G_{\sigma_{i}^{2}}\right)\left[G_{\delta_{i}^{\prime}}\left(1-G_{\delta_{i}^{\prime}}\right)+\frac{1}{2} G_{\delta_{i}^{\prime}}\left(2 G_{\sigma_{i}^{2}}+G_{\sigma_{i}^{4}}\right)\right] / n_{i}$. Thus, the $100(1-\zeta) \%$ FGCI for $\vartheta$ is

$$
C I_{\vartheta}^{(f g c i)}=\left[L_{\vartheta}^{(f g c i)}, U_{\vartheta}^{(f g c i)}\right]=\left[G_{\vartheta}(\zeta / 2), G_{\vartheta}(1-\zeta / 2)\right]
$$

where $G_{\vartheta}(\zeta)$ denotes the $\zeta^{\text {th }}$ percentiles of $G_{\vartheta}$. Algorithm 1 shows the computational steps for obtaining the FGCI.

\section{Algorithm 1: FGCI}

1) Generate $V_{i} \sim N(0,1)$ and $U_{i} \sim \chi_{n_{i(1)}-1}^{2}$ are independent.

2) Compute the FGPQs $G_{\mu_{i}}, G_{\sigma_{i}^{2}}$ and $G_{\delta_{i}^{\prime}}$.

3) Compute $G_{w_{i}}$ and $G_{\vartheta_{i}}$ leading to obtain $G_{\vartheta}$.

4) Repeat steps $1-3$, a number of times, $\mathrm{m}=2500$, compute $95 \% \mathrm{FGCI}$ for $\vartheta$, as given in (18).

\section{Large Sample Interval}

Recall that the Aitchitson estimator is $\hat{\vartheta}_{i}^{(A i t)}=\left(1-\hat{\delta}_{i}\right) \exp \left(\hat{\mu}_{i}\right) \psi_{n_{i(1)}}\left(\hat{\sigma}_{i}^{2} / 2\right)$ and the variance of $\hat{\vartheta}_{i}^{(A i t)}$ is $\operatorname{Var}\left[\hat{\vartheta}_{i}^{(A i t)}\right]=\exp \left(2 \mu_{i}+\sigma_{i}^{2}\right)\left[\delta_{i}\left(1-\delta_{i}\right)+\frac{1}{2}\left(1-\delta_{i}\right)\left(2 \sigma_{i}^{2}+\sigma_{i}^{4}\right)\right] / n_{i}$. The approximated variance is obtained by replacing $\hat{\mu}_{i}, \hat{\sigma}_{i}^{2}$ and $\hat{\delta}_{i}$. The pooled estimate of $\vartheta_{i}$ is given by

$$
\hat{\vartheta}=\frac{\sum_{i=1}^{k} w_{i} \hat{\vartheta}_{i}^{(A i t)}}{\sum_{i=1}^{k} w_{i}}
$$

where $w_{i}=1 / \widehat{\operatorname{Var}}\left[\hat{\vartheta}_{i}^{(A i t)}\right]$. Hence, the $100(1-\zeta) \%$ LS interval for $\vartheta$ is obtained as

$$
C I_{\vartheta}^{(l s)}=\left[L_{\vartheta}^{(l s)}, U_{\vartheta}^{(l s)}\right]=\left[\hat{\vartheta}-z_{1-\frac{\zeta}{2}} \sqrt{1 / \sum_{i=1}^{k} w_{i}}, \hat{\vartheta}+z_{1-\frac{\zeta}{2}} \sqrt{1 / \sum_{i=1}^{k} w_{i}}\right]
$$

where $z_{\zeta}$ denotes the $\zeta^{\text {th }}$ percentiles of standard normal $N(0,1)$. The LS interval can be estimated easily via Algorithm 2. 
Algorithm 2: LS

1) Compute $\hat{\vartheta}_{i}^{(A i t)}$ and $\widehat{\operatorname{Var}}\left[\hat{\vartheta}_{i}^{(A i t)}\right]$.

2) Compute $\hat{\vartheta}$.

3) Compute $95 \% \mathrm{LS}$ interval for $\vartheta$, as given in (20).

\section{Method of Variance Estimates Recovery}

This method produces a closed-form CI that is easy to compute. For this reason, the MOVER CI for the common delta-lognormal mean is considered for $k$ individual random samples. The MOVER for a linear combination of $\vartheta_{i} ; i=1,2, \ldots, k$ is as follows. Let $\hat{\vartheta}_{1}, \hat{\vartheta}_{2}, \ldots, \hat{\vartheta}_{k}$ be independent unbiased estimators of $\vartheta_{1}, \vartheta_{2}, \ldots, \vartheta_{k}$, respectively. In addition, let $\left[l_{i}, u_{i}\right]$ stand for the $100(1-\zeta) \% \mathrm{CI}$ for $\vartheta_{i}$. According to

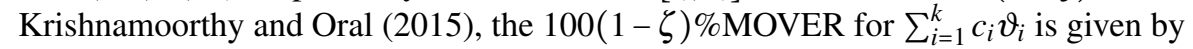

$$
\begin{aligned}
C I_{\sum_{i=1}^{k} c_{i} \vartheta_{i}} & =\left[L_{\sum_{i=1}^{k} c_{i} \vartheta_{i}}, U_{\sum_{i=1}^{k} c_{i} \vartheta_{i}}\right] \\
& =\left[\sum_{i=1}^{k} c_{i} \hat{\vartheta}_{i}-\sqrt{\sum_{i=1}^{k} c_{i}^{2}\left(\hat{\vartheta}_{i}-l_{i}^{*}\right)^{2}}, \sum_{i=1}^{k} c_{i} \hat{\vartheta}_{i}+\sqrt{\sum_{i=1}^{k} c_{i}^{2}\left(\hat{\vartheta}_{i}-u_{i}^{*}\right)^{2}}\right]
\end{aligned}
$$

where $l_{i}^{*}=\left\{\begin{array}{ll}l_{i} & ; c_{i}>0 \\ u_{i} & ; c_{i}<0\end{array}\right.$ and $u_{i}^{*}=\left\{\begin{array}{ll}u_{i} & ; c_{i}>0 \\ l_{i} & ; c_{i}<0\end{array}\right.$. Next, the closed-form CIs for $\vartheta_{i}$ are needed to construct MOVER for $\vartheta$. Thus, $\vartheta_{i}$ is $\log$-transformed as

$$
\ln \vartheta_{i}=\ln \delta_{i}^{*}+\left(\mu_{i}+\sigma_{i}^{2}\right)
$$

where $\delta_{i}^{*}=1-\delta_{i}$. Let $\hat{\mu}_{i}$, and $\hat{\sigma}_{i}^{2}$ and $\hat{\delta}^{*}$ be the unbiased estimates of $\mu_{i}, \sigma_{i}^{2}$, and $\delta_{i}$, respectively. The MOVER for a single delta-lognormal mean presented by Hasan and Krishnamoorthy (2018), the MOVER for $\vartheta_{i}$ is given by

$$
\begin{aligned}
& L_{\vartheta_{i}}=\exp \left\{\ln \hat{\delta}_{i}^{*}+\left(\hat{\mu}_{i}+\hat{\sigma}_{i}^{2}\right)-\sqrt{\left(\ln \hat{\delta}_{i}^{*}-l_{\ln \delta_{i}^{*}}\right)^{2}+\left(\hat{\mu}_{i}+\hat{\sigma}_{i}^{2}-l_{\mu_{i}+\sigma_{i}^{2}}\right)^{2}}\right\} \\
& U_{\vartheta_{i}}=\exp \left\{\ln \hat{\delta}_{i}^{*}+\left(\hat{\mu}_{i}+\hat{\sigma}_{i}^{2}\right)-\sqrt{\left(\ln \hat{\delta}_{i}^{*}-u_{\ln \delta_{i}^{*}}\right)^{2}+\left(\hat{\mu}_{i}+\hat{\sigma}_{i}^{2}-u_{\mu_{i}+\sigma_{i}^{2}}\right)^{2}}\right\}
\end{aligned}
$$

where

$$
\begin{aligned}
\left(l_{\ln \delta_{i}^{*}}, u_{\ln \delta_{i}^{*}}\right)= & \ln \left[\left(\hat{\delta}_{i}^{*}+\frac{T_{i, \zeta / 2}^{2}}{2 n_{i}} \mp T_{i, 1-\zeta / 2} \sqrt{\left.\left.\frac{\hat{\delta}_{i}^{*}\left(1-\hat{\delta}_{i}^{*}\right)}{n_{i}}+\frac{T_{i, \zeta / 2}^{2}}{4 n_{i}^{2}}\right) /\left(1+T_{i, \zeta / 2}^{2} / n_{i}\right)\right]}\right.\right. \\
\left(l_{\mu_{i}+\sigma_{i}^{2}}, u_{\mu_{i}+\sigma_{i}^{2}}\right)= & {\left[\left(\hat{\mu}_{i}+\hat{\sigma}_{i}^{2} / 2\right)-\left\{\left(\frac{Z_{i, \zeta / 2} \hat{\sigma}_{i}^{2}}{n_{i(1)}}\right)^{2}+\frac{\hat{\sigma}_{i}^{4}}{4}\left(1-\frac{n_{i(1)}-1}{\chi_{i, 1-\zeta / 2, n_{i(1)}-1}^{2}}\right)^{2}\right\}^{1 / 2},\right.} \\
& \left.\left(\hat{\mu}_{i}+\hat{\sigma}_{i}^{2} / 2\right)+\left\{\left(\frac{Z_{i, \zeta / 2} \hat{\sigma}_{i}^{2}}{n_{i(1)}}\right)^{2}+\frac{\hat{\sigma}_{i}^{4}}{4}\left(\frac{n_{i(1)}-1}{\chi_{i, \zeta / 2, n_{i(1)}-1}^{2}}-1\right)^{2}\right\}^{1 / 2}\right]
\end{aligned}
$$




$$
\begin{aligned}
C I_{\vartheta}^{(\text {mover })} & =\left[L_{\vartheta}, U_{\vartheta}\right] \\
& =\left[\frac{\sum_{i=1}^{k} w_{i} \hat{\vartheta}_{i}^{(A i t)}}{\sum_{i=1}^{k} w_{i}}-\sqrt{\frac{\sum_{i=1}^{k} w_{i}^{2}\left(\hat{\vartheta}_{i}^{(A i t)}-L_{\vartheta_{i}}\right)^{2}}{\sum_{i=1}^{k} w_{i}^{2}}}, \frac{\sum_{i=1}^{k} w_{i} \hat{\vartheta}_{i}^{(A i t)}}{\sum_{i=1}^{k} w_{i}}-\sqrt{\frac{\sum_{i=1}^{k} w_{i}^{2}\left(\hat{\vartheta}_{i}^{(A i t)}-U_{\vartheta_{i}}\right)^{2}}{\sum_{i=1}^{k} w_{i}^{2}}}\right]
\end{aligned}
$$

where $w_{i}=1 / \widehat{\operatorname{Var}}\left[\hat{\vartheta}_{i}^{(\text {Ait })}\right]$. Algorithm 3 describes the steps to construct the MOVER interval.

\section{Algorithm 3: MOVER}

1) Compute CIs for $\ln \delta_{i}^{*}$ and $\mu_{i}+\sigma_{i}^{2}$ are $\left(l_{\ln \delta_{i}^{*}}, u_{\ln \delta_{i}^{*}}\right)$ and $\left(l_{\mu_{i}+\sigma_{i}^{2}}, u_{\mu_{i}+\sigma_{i}^{2}}\right)$, respectively.

2) Compute MOVER for $\vartheta_{i}$, as given in (23).

3) Compute 95\% MOVER for $\vartheta$, given in (25).

\section{Parametric Bootstrap}

This is developed from the parametric bootstrap on the common mean of several heterogeneous lognormal distributions, proposed by Malekzadeh and Kharrati-Kopaei (2019). The delta-lognormal mean is transformed by taking the logarithm as

$$
\mu_{i}=\ln \left(\frac{\vartheta}{1-\delta_{i}}\right)-\frac{\sigma_{i}^{2}}{2}
$$

The likelihood of $\left(\vartheta, \sigma_{i}^{2}, \delta_{i}\right)$ is

$$
L\left(\vartheta, \sigma_{i}^{2}, \delta_{i} \mid w_{i j}\right)=\prod_{i=1}^{k}\left(\begin{array}{c}
n_{i} \\
n_{i(0)}
\end{array}\right) \delta_{i}\left(1-\delta_{i}\right) \frac{1}{\left(2 \pi \sigma_{i}^{2}\right)^{n_{i(1)} / 2}} \exp \left\{-\frac{1}{2 \sigma_{i}^{2}} \sum_{j=1}^{n_{i(1)}}\left(\ln w_{i j}-\ln \left(\frac{\vartheta}{1-\delta_{i}}\right)+\frac{\sigma_{i}^{2}}{2}\right)^{2}\right\}
$$

which enables obtaining the maximum likelihood estimates of $\ln \vartheta$ and $\sigma_{i}^{2}$ as

$$
\begin{aligned}
\ln \hat{\vartheta}_{m l e} & =\frac{\sum_{i=1}^{k} \hat{w}_{m l e, i}\left[\hat{\mu}_{i}+\ln \left(1-\hat{\delta}_{i}\right)\right]+N / 2}{\sum_{i=1}^{k} \hat{w}_{m l e, i}} \\
\hat{\sigma}_{m l e, i}^{2} & =-2+2 \sqrt{1+\hat{\sigma}_{i}^{2}+\left\{\hat{\mu}-\ln \left[\hat{\vartheta} /\left(1-\hat{\delta}_{i}\right)\right]\right\}^{2}}
\end{aligned}
$$

where $\hat{w}_{m l e, i}=n_{i(1)} / \hat{\sigma}_{m l e, i}^{2}$ and $\ln \hat{\vartheta}_{=} \frac{\sum_{i=1}^{k} \hat{w}_{i}\left[\hat{\mu}_{i}+\ln \left(1-\hat{\delta}_{i}\right)\right]+N / 2}{\sum_{i=1}^{k} \hat{w}_{i}} ; \hat{w}_{i}=n_{i(1)} / \hat{\sigma}_{i}^{2}$. If $\delta_{i}=0$, then it becomes the common lognormal mean (see Krishnamoorthy and Oral (2015) for a detailed explanation). By applying central limit theorem, we obtain $\left(\ln \hat{\vartheta}_{m l e}-\ln \vartheta\right) \sqrt{\sum_{i=1}^{k} \hat{w}_{m l e, i}} \sim N(0,1)$ such that $T=\left(\ln \hat{\vartheta}_{m l e}-\ln \vartheta\right)^{2} \sum_{i=1}^{k} \hat{w}_{m l e, i} \sim$ $\chi_{n_{i(1)}-1}^{2}$. It is well-known that $\hat{\mu}_{i}, \hat{\sigma}_{i}^{2}$ and $\hat{\delta}_{i}$ are independent random variables for which $\hat{\mu}_{i} \sim N\left(\ln \left(\frac{\vartheta}{1-\delta_{i}}\right)-\right.$ $\left.\frac{\sigma_{i}^{2}}{2}, \sigma_{i}^{2} / n_{i(1)}\right),\left(n_{i(1)}-1\right) \hat{\sigma}_{i}^{2} / \sigma_{i}^{2} \sim \chi_{n_{i(1)}}^{2}$ and $\hat{\delta}_{i} \sim N\left(\delta, \delta(1-\delta) / n_{i}\right)$ are obtained, respectively. Let $\eta=\mu_{i}+\sigma_{i}^{2} / 2$ so that we can write $T=\frac{\sum_{i=1}^{k} \hat{w}_{m l e, i}\left[\hat{\mu}_{i}+\ln \left(1-\hat{\delta}_{i}\right)-\eta-\ln \left(1-\delta_{i}\right)\right]+N / 2}{\sum_{i=1}^{k} \hat{w}_{m l e, i}}$. It can be seen that the distribution of $T$ is complicated, possibly depending on nuisance parameters $\sigma_{i}^{2}$ and $\delta_{i}$, but not on $\ln \vartheta$. Thus, the exact distribution of $\mathrm{T}$ is unknown in practice, and so we propose the PB pivotal variable corresponding to $T^{P B}$ as

$$
T^{P B}=\left(\ln \hat{\vartheta}_{m l e}^{P B}-\ln \hat{\vartheta}\right)^{2} \sum_{i=1}^{k} \hat{w}_{m l e, i}^{P B}
$$


where $\ln \hat{\vartheta}_{m l e}^{P B}=\frac{\sum_{i=1}^{k} \hat{w}_{m l e, i}^{P B}\left[\hat{\mu}_{i}^{P B}+\ln \left(1-\hat{\delta}_{i}^{P B}\right)\right]+N / 2}{\sum_{i=1}^{k} \hat{w}_{m l e, i}^{P B}}, \hat{w}_{i}^{P B}=n_{i(1)} / \hat{\sigma}_{i}^{2 B}, \hat{\mu}_{i}^{P B} \sim N\left(\hat{\mu}_{i}^{B}, \hat{\sigma}_{i}^{2 B} / n_{i(1)}\right), \hat{\sigma}_{i}^{2 P B} \sim \hat{\sigma}_{i}^{B 2} \chi_{n_{i(1)}}^{2}$ $/\left(n_{i(1)}-1\right)$ and $\hat{\delta}^{P B} \sim \operatorname{beta}\left(n_{i(0)}^{B}+0.5, n_{i(1)}^{B}+0.5\right), n_{i(0)}^{B}=n_{i} \hat{\delta}_{i}^{B}$, and $n_{i(1)}^{B}=n_{i}-n_{i(0)}^{B}$. Note that $\hat{\mu}_{i}^{B}, \hat{\sigma}_{i}^{2 B}$, and $\hat{\delta}_{i}^{B}$ are the observed values of $\hat{\mu}_{i}, \hat{\sigma}_{i}^{2}$, and $\hat{\delta}_{i}$, respectively, from random sampling with replacement based on the bootstrap approach. Thus, the $100(1-\zeta) \% \mathrm{~PB}$ interval for $\vartheta$ is given by

$$
C I_{\vartheta}^{(p b)}=\exp \left[\ln \hat{\vartheta}_{m l e} \mp \sqrt{q_{\zeta}^{P B} / \sum_{i=1}^{k} \hat{w}_{m l e, i}}\right]
$$

where $q_{\zeta}^{P B}$ denotes the $(1-\zeta)^{t h}$ percentile of distribution of $T^{P B}$. The PB interval can be constructed as shown in Algorithm 4.

\section{Algorithm 4: PB}

1) Compute $\hat{\mu}_{i}, \hat{\sigma}_{i}^{2}$ and $\hat{\delta}$ leading to obtain $\ln \hat{\vartheta}$.

2) Compute $\ln \hat{\vartheta}_{m l e}$ and $\hat{\sigma}_{m l e . i}^{2}$.

3) Generate $\hat{\mu}_{i}^{P B}, \hat{\sigma}_{i}^{2 P B}$ and $\hat{\delta}_{i}^{P B}$ leading to compute $\ln \hat{\vartheta}_{m l e}^{P B}$.

4) Repeat steps $1-3$, a number of time $m=2500$, compute $T^{P B}$ to obtain $q_{\zeta}^{P B}$.

5) Compute $95 \% \mathrm{~PB}$ interval for $\vartheta$, as given in (30).

\section{Highest Posterior Density Intervals}

The HPD interval is constructed from the posterior distribution, as defined by Box and Tiao (1973). Note that the prior of $\vartheta_{i}$ is updated with its likelihood function thereby obtaining the posterior distribution based on the Bayesian approach. Recall that $W_{i j} \sim \Delta\left(\mu_{i}, \sigma_{i}^{2}, \delta_{i}\right)$, then the likelihood is given by

$$
P\left(w_{i j} \mid \mu_{i}, \sigma_{i}^{2}, \delta_{i}\right) \propto \prod_{i=1}^{k} \delta_{i}^{n_{i(0)}}\left(1-\delta_{i}\right)^{n_{i(1)}}\left(\sigma_{i}^{2}\right)^{-n_{i(1)} / 2} \exp \left\{-\frac{1}{2 \sigma_{i}^{2}} \sum_{j=1}^{n_{i(1)}}\left(\ln w_{i j}-\mu_{i}\right)^{2}\right\}
$$

For $k$ individual samples, Miroshnikov et al. (2015) described the pooled independent sub-posterior samples toward the joint posterior distributions $\vartheta$ are combined using weighted averages as follows:

$$
\vartheta^{\text {post }}=\sum_{i=1}^{k} w_{i} \vartheta_{i}^{\text {post }}\left(\sum_{i=1}^{k} w_{i}\right)^{-1}
$$

where $\vartheta_{i}^{\text {post }}$ are the posterior samples of $\vartheta_{i}$, for $i=1,2, \ldots, k$. The inverse of the sample variance is used to weight the posterior based on the $i^{t h}$ samples is denoted as $w_{i}=\operatorname{Var}^{-1}\left(\hat{\vartheta}_{i} \mid w_{i j}\right)$. Different priors have been developed for estimating the common delta-lognormal mean, two of which are derived in the following subsections.

\section{Jeffreys' Rule Prior}

Harvey and van der Merwe (2012) defined this prior as

$$
P(\vartheta)_{J R} \propto \prod_{i=1}^{k} \sigma_{i}^{-3} \delta_{i}^{-1 / 2}\left(1-\delta_{i}\right)^{1 / 2}
$$

which is combined with the likelihood (34) to obtain the posterior of $\vartheta$ as

$$
\begin{aligned}
P\left(w_{i j} \mid \vartheta\right) \propto & \prod_{i=1}^{k} \delta_{i}^{n_{i(0)}-1 / 2}\left(1-\delta_{i}\right)^{n_{i(1)}+1 / 2}\left(\sigma_{i}^{2}\right)^{-\left(n_{i(1)}+3\right) / 2} \exp \left\{-\frac{1}{2 \sigma_{i}^{2}} \sum_{j=1}^{n_{i(1)}}\left(\ln w_{i j}-\mu_{i}\right)^{2}\right\} \\
\propto & \prod_{i=1}^{k} \delta_{i}^{\left(n_{i(0)}+1 / 2\right)-1}\left(1-\delta_{i}\right)^{\left(n_{i(1)}+3 / 2\right)-1}\left(\sigma_{i}^{2}\right)^{-\frac{\left(n_{i(1)}+1\right)}{2}-1} \\
& \exp \left\{-\frac{1}{2 \sigma_{i}^{2}}\left[\left(n_{i(1)}-1\right) \hat{\sigma}_{i}^{2}+n_{i(1)}\left(\hat{\mu}_{i}-\mu_{i}\right)^{2}\right]\right\}
\end{aligned}
$$


This leads to obtaining the marginal posterior distributions of $\mu_{i}, \sigma_{i}^{2}$ and $\delta_{i}$ as

$$
\begin{aligned}
\mu_{i}^{(J R)} \mid \sigma_{i, J R}^{2}, w_{i j} & \sim N\left(\hat{\mu}_{i}, \sigma_{i}^{2(J R)} / n_{i(1)}\right) \\
\sigma_{i}^{2(J R)} \mid w_{i j} & \sim I G\left(\left(n_{i(1)}+1\right) / 2,\left(n_{i(1)}+1\right) \hat{\sigma}_{i}^{2} / 2\right) \\
& \delta_{i}^{(J R)} \mid w_{i j} \sim \operatorname{beta}\left(n_{i(0)}+1 / 2, n_{i(1)}+3 / 2\right)
\end{aligned}
$$

The pooled posterior of $\vartheta$ is weighted by its inversely estimated variance as follows:

$$
\vartheta^{p o s t}=\sum_{i=1}^{k} w_{i}^{(J R)} \vartheta_{i}^{(J R)_{p}}\left(\sum_{i=1}^{k} w_{i}^{(J R)}\right)^{-1}
$$

where

$$
\begin{aligned}
& \vartheta_{i}^{(J R)_{p}}=\left(1-\delta_{i}^{(J R)}\right) \exp \left(\mu_{i}^{(J R)}+\sigma_{i}^{2(J R)} / 2\right) \\
& w_{i}^{(J R)}=\left\{n_{i}^{-1} \exp \left(2 \mu_{i}^{(J R)}+\sigma_{i}^{2(J R)}\right)\left[\delta_{i}^{(J R)}\left(1-\delta_{i}^{(J R)}\right)+\frac{1}{2}\left(1-\delta_{i}^{(J R)}\right)\left(2 \sigma_{i}^{2(J R)}+\sigma_{i}^{4(J R)}\right)\right]\right\}^{-1}
\end{aligned}
$$

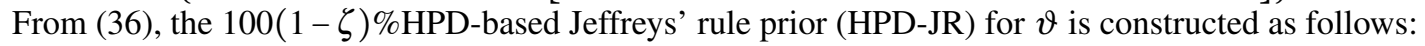

\section{Normal-Gamma-Beta Prior}

Maneerat et al. (2020) proposed a HPD based on the normal-gamma prior for the ratio of delta-lognormal variances that worked better than the HPD-JR of Harvey and van der Merwe (2012). Suppose that $\boldsymbol{Y}=\ln \boldsymbol{W}$ be a random variable of normal distribution with mean $\boldsymbol{\mu}=\left(\mu_{1}, \mu_{2}, \ldots, \mu_{k}\right)$ and precision $\boldsymbol{\lambda}=\left(\lambda_{1}, \lambda_{2}, \ldots, \lambda_{k}\right)$ where $\boldsymbol{W} \sim L N(\boldsymbol{\mu}, \boldsymbol{\lambda})$ and $\lambda_{i}=\sigma_{i}^{-2}$. The HPD-based normal-gamma-beta prior (HPDNGB) of $\vartheta=\left(\mu_{i}, \lambda_{i}, \delta_{i}\right)^{\prime}$ is defined as

$$
P(\vartheta) \propto \prod_{i=1}^{k} \lambda_{i}^{-1}\left[\delta_{i}\left(1-\delta_{i}\right)\right]^{-1 / 2}
$$

where $\left(\mu_{i}, \lambda_{i}\right)$ follows a normal-gamma distribution, and $\delta_{i}$ follows a beta distribution, denoted as $\left(\mu_{i}, \lambda_{i}\right) \sim N G\left(\mu_{i}, \lambda_{i} \mid \mu, k_{i(0)}=0, \alpha_{i(0)}=-1 / 2, \beta_{i(0)}=0\right)$ and $\delta_{i} \sim \operatorname{beta}(1 / 2,1 / 2)$, respectively. When the the prior (37) is combined with the likelihood (34), then the posterior density of $\vartheta$ becomes

$$
\begin{aligned}
P\left(\vartheta \mid w_{i j}\right) \propto & \prod_{i=1}^{k} \delta_{i}^{n_{i(0)}-1 / 2}\left(1-\delta_{i}\right)^{n_{i(1)}-1 / 2} \lambda_{i}^{\frac{n_{i(1)}-1}{2}-1} \exp \left\{-\frac{\lambda_{i}}{2} \sum_{j=1}^{n_{i(1)}}\left(\ln w_{i j}-\hat{\mu}_{i}\right)^{2}\right\} \lambda_{i}^{1 / 2} \\
& \exp \left\{-\frac{n_{i(1)} \lambda_{i}}{2}\left(\mu_{i}-\mu_{i}^{*}\right)^{2}\right\}
\end{aligned}
$$

which can be integrated out to obtain the marginal posterior distributions of $\mu_{i}, \lambda_{i}$ and $\delta_{i}$ as follows:

$$
\begin{aligned}
& \mu_{i}^{(N G B)} \mid w_{i j} \sim t_{d f}\left(\mu_{i} \mid \hat{\mu}_{i}, \sum_{j=1}^{n_{i(1)}}\left(\ln w_{i j}-\hat{\mu}_{i}\right)^{2} /\left[n_{i(1)}\left(n_{i(1)}-1\right)\right]\right) \\
& \lambda_{i}^{(N G B)} \mid w_{i j} \sim G\left(\lambda_{i} \mid\left(n_{i(1)}-1\right) / 2, \sum_{j=1}^{n_{i(1)}}\left(\ln w_{i j}-\hat{\mu}_{i}\right)^{2} / 2\right) \\
& \delta_{i}^{(N G B)} \mid w_{i j} \sim \operatorname{beta}\left(n_{i(0)}+1 / 2, n_{i(1)}+1 / 2\right)
\end{aligned}
$$

where $d f=2\left(n_{i(1)}-1\right)$ and $\sigma_{i}^{2(N G B)} \mid w_{i j} \sim I G\left(\sigma_{i}^{2} \mid\left(n_{i(1)}-1\right) / 2, \sum_{j=1}^{n_{i(1)}}\left(\ln w_{i j}-\hat{\mu}_{i}\right)^{2} / 2\right)$. Similarly, the pooled posterior of $\vartheta$ is given by

$$
\vartheta^{p o s t}=\sum_{i=1}^{k} w_{i}^{(N G B)} \vartheta_{i}^{(N G B)_{p}}\left(\sum_{i=1}^{k} w_{i}^{(N G B)}\right)^{-1}
$$


where

$$
\begin{aligned}
& \vartheta_{i}^{(N G B)_{p}}=\left(1-\delta_{i}^{(N G B)}\right) \exp \left(\mu_{i}^{(N G B)}+\sigma_{i}^{2(N G B)} / 2\right) \\
& w_{i}^{(N G B)}=\left\{n_{i}^{-1} \exp \left(2 \mu_{i}^{(N G B)}+\sigma_{i}^{2(N G B)}\right)\left[\delta_{i}^{(N G B)}\left(1-\delta_{i}^{(N G B)}\right) \frac{1}{2}\left(1-\delta_{i}^{(N G B)}\right)\left(2 \sigma_{i}^{2(N G B)}+\sigma_{i}^{4(N G B)}\right)\right]\right\}^{-1}
\end{aligned}
$$

Hence, the $100(1-\zeta) \%$ HPD-HGB for $\vartheta$ is constructed in (40). Algorithm 5 details the steps to construct the HPD-JR and HPD-NGB.

\section{Algorithm 5: HPD-JR and HPD-NGB}

1) Compute $\hat{\mu}_{i}, \hat{\sigma}_{i}^{2}$ and $\hat{\delta}$.

2) Generate the posterior densities of $\mu_{i}, \sigma_{i}^{2}$ and $\delta_{i}$ based-Jeffreys' rule (JR) and normal-gamma-beta (NGB) priors, as given in (35) and (39), respectively.

3) Compute the pooled posterior of $\vartheta$ based on JR and NGB priors, as given in (36) and (40), respectively.

4) Compute 95\%HPD-JR and HPD-NGB for $\vartheta$, defined by Box and Tiao (1973).

\section{SIMULATION STUDIES AND RESULTS}

The performances of the CIs were assessed by comparing their coverage probabilities (CPs) and average length (ALs) using Monte Carlo simulation. The best-performing $\mathrm{CI}$ is the one where the $\mathrm{CP}$ is closest to or greater than the nominal confidence level $1-\zeta$ while also having an AL with the narrowest width. The CIs for the common delta-lognormal mean constructed using FGCI, LS, MOVER, PB, HPD-JR, and HPD-NGB were assessed in the study, the parameter settings for which are provided in Table 1. The number of generated random samples was fixed at $M=5000$. For FGCI, the number of FGPQs was $Q=2500$ for each set of 5000 random samples. Algorithm 6 shows the computational steps to estimate the $\mathrm{CP}$ and AL performances of all of the methods.

\begin{tabular}{|c|c|c|c|}
\hline Scenarios & $\left(n_{1}, \ldots, n_{k}\right)$ & $\left(\delta_{1}, \ldots, \delta_{k}\right)$ & $\left(\sigma_{1}^{2}, \ldots, \sigma_{k}^{2}\right)$ \\
\hline \multicolumn{4}{|l|}{$k=2$} \\
\hline $1-9$ & $\left(30_{2}\right)$ & $(0.1,0.2),(0.2,0.5),(0.3,0.7)$ & $(1,2),(2,4),(3,5)$ \\
\hline $10-18$ & $(30,50)$ & $(0.1,0.2),(0.2,0.5),(0.3,0.7)$ & $(1,2),(2,4),(3,5)$ \\
\hline $19-27$ & $\left(50_{2}\right)$ & $(0.1,0.2),(0.2,0.5),(0.3,0.7)$ & $(1,2),(2,4),(3,5)$ \\
\hline $28-36$ & $(50,100)$ & $(0.1,0.2),(0.2,0.5),(0.3,0.7)$ & $(1,2),(2,4),(3,5)$ \\
\hline $37-45$ & $\left(100_{2}\right)$ & $(0.1,0.2),(0.2,0.5),(0.3,0.7)$ & $(1,2),(2,4),(3,5)$ \\
\hline \multicolumn{4}{|l|}{$k=5$} \\
\hline $46-54$ & $\left(30_{5}\right)$ & $\left(0.05,0.1_{2}, 0.2_{2}\right),\left(0.2_{2}, 0.4_{3}\right),\left(0.5_{2}, 0.7_{3}\right)$ & $\left(1_{2}, 2_{3}\right),\left(2_{2}, 3_{3}\right),\left(3_{2}, 5_{3}\right)$ \\
\hline $55-63$ & $\left(30_{2}, 50_{3}\right)$ & $\left(0.05,0.1_{2}, 0.2_{2}\right),\left(0.2_{2}, 0.4_{3}\right),\left(0.5_{2}, 0.7_{3}\right)$ & $\left(1_{2}, 2_{3}\right),\left(2_{2}, 3_{3}\right),\left(3_{2}, 5_{3}\right)$ \\
\hline $64-72$ & $\left(30_{2}, 50_{2}, 100\right)$ & $\left(0.05,0.1_{2}, 0.2_{2}\right),\left(0.2_{2}, 0.4_{3}\right),\left(0.5_{2}, 0.7_{3}\right)$ & $\left(1_{2}, 2_{3}\right),\left(2_{2}, 3_{3}\right),\left(3_{2}, 5_{3}\right)$ \\
\hline $73-81$ & $\left(30,50_{2}, 100_{2}\right)$ & $\left(0.05,0.1_{2}, 0.2_{2}\right),\left(0.2_{2}, 0.4_{3}\right),\left(0.5_{2}, 0.7_{3}\right)$ & $\left(1_{2}, 2_{3}\right),\left(2_{2}, 3_{3}\right),\left(3_{2}, 5_{3}\right)$ \\
\hline $82-90$ & $\left(50_{5}\right)$ & $\left(0.05,0.1_{2}, 0.2_{2}\right),\left(0.2_{2}, 0.4_{3}\right),\left(0.5_{2}, 0.7_{3}\right)$ & $\left(1_{2}, 2_{3}\right),\left(2_{2}, 3_{3}\right),\left(3_{2}, 5_{3}\right)$ \\
\hline $91-99$ & $\left(50_{2}, 100_{3}\right)$ & $\left(0.05,0.1_{2}, 0.2_{2}\right),\left(0.2_{2}, 0.4_{3}\right),\left(0.5_{2}, 0.7_{3}\right)$ & $\left(1_{2}, 2_{3}\right),\left(2_{2}, 3_{3}\right),\left(3_{2}, 5_{3}\right)$ \\
\hline $100-108$ & $\left(100_{5}\right)$ & $\left(0.05,0.1_{2}, 0.2_{2}\right),\left(0.2_{2}, 0.4_{3}\right),\left(0.5_{2}, 0.7_{3}\right)$ & $\left(1_{2}, 2_{3}\right),\left(2_{2}, 3_{3}\right),\left(3_{2}, 5_{3}\right)$ \\
\hline \multicolumn{4}{|c|}{ ( } \\
\hline $109-114$ & $\left(30_{5}, 50_{5}\right)$ & $\left(0.1_{5}, 0.2_{5}\right),\left(0.2_{5}, 0.5_{5}\right)$ & $\left(1_{5}, 2_{5}\right),\left(22_{5}, 4_{5}\right),\left(3_{5}, 5_{5}\right)$ \\
\hline $115-120$ & $\left(30_{3}, 50_{3}, 100_{4}\right)$ & $\left(0.1_{5}, 0.2_{5}\right),\left(0.2_{5}, 0.5_{5}\right)$ & $\left(1_{5}, 2_{5}\right),\left(2_{5}, 4_{5}\right),\left(3_{5}, 5_{5}\right)$ \\
\hline $121-126$ & $\left(50_{5}, 100_{5}\right)$ & $\left(0.1_{5}, 0.2_{5}\right),\left(0.2_{5}, 0.5_{5}\right)$ & $\left(1_{5}, 2_{5}\right),\left(2_{5}, 4_{5}\right),\left(3_{5}, 5_{5}\right)$ \\
\hline
\end{tabular}

Table 1. Parameter settings for sample cases $k=2,5,10$.

Note: $\left(30_{5}\right)$ stands for $(30,30,30,30,30)$.

\section{Algorithm 6: Comparison of CPs and ALs for all Cls.}

For $g=1$ to $M$. Generate $w_{i j} \sim \Delta\left(\mu_{i}, \sigma_{i}^{2}, \delta_{i}\right)$.

Compute the unbiased estimates $\hat{\mu}_{i}, \hat{\sigma}_{i}^{2}$ and $\hat{\delta}$.

Compute the 95\%CIs for $\vartheta$ based on FGCI, LS, MOVER, PB and the HPDs via Algorithms 1, 2, 3, 4 and 5 , respectively.

Let $A_{g}=1$ if $\vartheta$ falls within the intervals of FGCI, LS, MOVER, PB or the HPDs, else $A_{g}=0$.

The $\mathrm{CP}$ and $\mathrm{AL}$ for each method are obtained by $\mathrm{CP}=(1 / M) \sum_{g=1}^{M} A_{g}$ and $\mathrm{AL}=(U-L) / M$, respectively, where $U$ and $L$ are the upper and lower confidence limits, respectively. (end $g$ loop) 
The numerical results for the $\mathrm{CI}$ performances are presented in terms of $\mathrm{CP}$ and $\mathrm{AL}$ for various sample cases. For $k=2$ (Table 2 and Figure 1), FGCI performed well for small-to-moderate sample sizes, as well as for large $\sigma_{i}^{2}$ and a moderate-to-large sample size. HPD-NGB attained stable and the best CP and AL values for small $\sigma_{i}^{2}$ and a moderate-to-large sample size. MOVER and PB attained correct CPs but wider ALs than the other methods whereas LS and HPD-JR had lower CPs and narrower ALs. For $k=5$ (Table 3 and Figure 2), there were only two methods producing better CPs than the other methods in the various situations: MOVER (small $\delta_{i}$ and $\sigma_{i}^{2}$ ) and PB (large $\delta_{i}$ and $\sigma_{i}^{2}$ ). Moreover, the results were similar for $k=10$ (Table 4 and Figure 3).

As previously mentioned, our findings show that FGCI works well for small sample case because the FGPQ of $\sigma_{i}^{2}$ might contain some weak points that affect the FGPQ of $\mu_{i}$ as the sample case increases. For large sample sizes, MOVER was the best method for small $\sigma^{2}$, which is possibly caused by the CI for $\mu_{i}+\sigma_{i}^{2}$. Meanwhile, the next best one was PB, which has the strong point of using a resampling technique to collect information about several populations even when the variance $\sigma^{2}$ is large.

Table 2. Performance measures of $95 \%$ CIs for $\vartheta: 2$ sample cases

\begin{tabular}{|c|c|c|c|c|c|c|c|c|c|c|c|c|}
\hline \multirow{2}{*}{ Scenarios } & \multicolumn{6}{|l|}{$\mathrm{CP}$} & \multicolumn{6}{|l|}{$\mathrm{AL}$} \\
\hline & FG & LS & MO & $\mathrm{PB}$ & HJ & $\mathrm{HN}$ & FG & LS & MO & $\mathrm{PB}$ & HJ & $\mathrm{HN}$ \\
\hline \multicolumn{13}{|l|}{$k=2$} \\
\hline 1 & 0.959 & 0.897 & 0.967 & 0.994 & 0.916 & 0.941 & 1.556 & 1.296 & 2.005 & 2.324 & 1.353 & 1.436 \\
\hline 2 & 0.958 & 0.857 & 0.947 & 0.996 & 0.924 & 0.941 & 5.169 & 3.770 & 7.287 & 8.631 & 4.186 & 4.335 \\
\hline 3 & 0.963 & 0.821 & 0.959 & 0.996 & 0.919 & 0.932 & 13.088 & 8.675 & 23.312 & 22.883 & 9.905 & 10.220 \\
\hline 4 & 0.962 & 0.886 & 0.978 & 0.995 & 0.917 & 0.939 & 1.487 & 1.211 & 2.181 & 2.155 & 1.247 & 1.386 \\
\hline 5 & 0.953 & 0.832 & 0.962 & 0.995 & 0.913 & 0.922 & 4.875 & 3.487 & 9.881 & 7.818 & 3.811 & 4.066 \\
\hline 6 & 0.951 & 0.793 & 0.971 & 0.991 & 0.901 & 0.912 & 12.311 & 7.740 & 37.615 & 21.129 & 8.875 & 9.378 \\
\hline 7 & 0.961 & 0.829 & 0.972 & 0.982 & 0.920 & 0.940 & 1.511 & 1.095 & 3.968 & 2.173 & 1.224 & 1.406 \\
\hline 8 & 0.950 & 0.778 & 0.974 & 0.995 & 0.900 & 0.911 & 4.821 & 3.123 & 293.620 & 7.649 & 3.566 & 3.916 \\
\hline 9 & 0.939 & 0.725 & 0.973 & 0.988 & 0.866 & 0.887 & 13.159 & 7.067 & $8.0 \mathrm{e} 4$ & 23.632 & 8.680 & 9.419 \\
\hline 10 & 0.960 & 0.900 & 0.965 & 0.992 & 0.915 & 0.941 & 1.503 & 1.249 & 1.936 & 2.225 & 1.362 & 1.395 \\
\hline 11 & 0.961 & 0.848 & 0.941 & 0.992 & 0.924 & 0.940 & 5.128 & 3.712 & 6.765 & 8.667 & 4.298 & 4.368 \\
\hline 12 & 0.965 & 0.819 & 0.952 & 0.998 & 0.919 & 0.931 & 12.297 & 8.382 & 20.057 & 21.597 & 9.819 & 9.894 \\
\hline 13 & 0.960 & 0.896 & 0.977 & 0.992 & 0.917 & 0.942 & 1.366 & 1.147 & 1.909 & 2.004 & 1.203 & 1.271 \\
\hline 14 & 0.961 & 0.851 & 0.964 & 0.996 & 0.916 & 0.931 & 4.593 & 3.422 & 7.236 & 7.458 & 3.761 & 3.889 \\
\hline 15 & 0.949 & 0.790 & 0.958 & 0.994 & 0.894 & 0.905 & 11.116 & 7.517 & 22.293 & 19.310 & 8.507 & 8.718 \\
\hline 16 & 0.963 & 0.860 & 0.972 & 0.974 & 0.928 & 0.943 & 1.354 & 1.033 & 2.141 & 1.928 & 1.155 & 1.257 \\
\hline 17 & 0.952 & 0.803 & 0.976 & 0.992 & 0.900 & 0.917 & 4.397 & 3.048 & 10.772 & 6.889 & 3.418 & 3.630 \\
\hline 18 & 0.940 & 0.737 & 0.968 & 0.989 & 0.872 & 0.889 & 11.065 & 6.663 & 43.755 & 19.011 & 7.903 & 8.247 \\
\hline 19 & 0.961 & 0.914 & 0.966 & 0.992 & 0.921 & 0.946 & 1.153 & 1.009 & 1.382 & 1.696 & 1.043 & 1.076 \\
\hline 20 & 0.965 & 0.895 & 0.946 & 0.991 & 0.938 & 0.949 & 3.668 & 2.924 & 4.309 & 5.981 & 3.178 & 3.229 \\
\hline 21 & 0.962 & 0.863 & 0.952 & 0.996 & 0.930 & 0.940 & 8.747 & 6.665 & 11.805 & 14.651 & 7.272 & 7.395 \\
\hline 22 & 0.958 & 0.910 & 0.978 & 0.985 & 0.919 & 0.944 & 1.091 & 0.945 & 1.414 & 1.555 & 0.945 & 1.031 \\
\hline 23 & 0.965 & 0.883 & 0.969 & 0.996 & 0.926 & 0.937 & 3.336 & 2.695 & 4.578 & 5.204 & 2.811 & 2.950 \\
\hline 24 & 0.961 & 0.840 & 0.972 & 0.995 & 0.921 & 0.928 & 7.887 & 5.987 & 13.164 & 12.757 & 6.338 & 6.605 \\
\hline 25 & 0.969 & 0.868 & 0.980 & 0.958 & 0.930 & 0.953 & 1.120 & 0.866 & 1.610 & 1.503 & 0.937 & 1.070 \\
\hline 26 & 0.954 & 0.839 & 0.970 & 0.997 & 0.916 & 0.926 & 3.208 & 2.433 & 6.544 & 4.735 & 2.621 & 2.830 \\
\hline 27 & 0.946 & 0.773 & 0.970 & 0.992 & 0.893 & 0.903 & 7.803 & 5.443 & 26.105 & 12.382 & 6.011 & 6.376 \\
\hline 28 & 0.958 & 0.912 & 0.972 & 0.979 & 0.916 & 0.947 & 1.119 & 0.952 & 1.397 & 1.615 & 1.054 & 1.051 \\
\hline 29 & 0.956 & 0.872 & 0.921 & 0.958 & 0.927 & 0.943 & 3.745 & 2.836 & 4.238 & 6.098 & 3.338 & 3.330 \\
\hline 30 & 0.961 & 0.846 & 0.937 & 0.987 & 0.925 & 0.936 & 8.488 & 6.274 & 10.833 & 13.94 & 7.332 & 7.320 \\
\hline 31 & 0.962 & 0.927 & 0.985 & 0.978 & 0.919 & 0.949 & 0.984 & 0.876 & 1.322 & 1.4 & 0.908 & 0.929 \\
\hline 32 & 0.960 & 0.880 & 0.958 & 0.992 & 0.925 & 0.940 & 3.214 & 2.618 & 4.169 & 5.150 & 2.818 & 2.860 \\
\hline 33 & 0.958 & 0.838 & 0.960 & 0.994 & 0.910 & 0.925 & 7.360 & 5.744 & 10.824 & 12.105 & 6.256 & 6.279 \\
\hline 34 & 0.963 & 0.888 & 0.977 & 0.922 & 0.938 & 0.954 & 0.975 & 0.785 & 1.322 & 1.352 & 0.876 & 0.922 \\
\hline 35 & 0.958 & 0.860 & 0.971 & 0.995 & 0.917 & 0.929 & 2.915 & 2.343 & 4.321 & 4.424 & 2.486 & 2.586 \\
\hline 36 & 0.951 & 0.820 & 0.973 & 0.995 & 0.901 & 0.916 & 6.726 & 5.103 & 11.951 & 10.823 & 5.511 & 5.626 \\
\hline 37 & 0.957 & 0.935 & 0.960 & 0.970 & 0.927 & 0.948 & 0.802 & 0.722 & 0.923 & 1.168 & 0.743 & 0.753 \\
\hline 38 & 0.955 & 0.916 & 0.926 & 0.953 & 0.942 & 0.948 & 2.442 & 2.044 & 2.541 & 3.935 & 2.220 & 2.219 \\
\hline 39 & 0.957 & 0.888 & 0.939 & 0.981 & 0.937 & 0.945 & 5.608 & 4.594 & 6.295 & 9.049 & 4.984 & 4.998 \\
\hline 40 & 0.961 & 0.942 & 0.975 & 0.957 & 0.924 & 0.954 & 0.740 & 0.679 & 0.911 & 1.062 & 0.659 & 0.702 \\
\hline 41 & 0.961 & 0.920 & 0.960 & 0.988 & 0.933 & 0.950 & 2.199 & 1.925 & 2.558 & 3.401 & 1.958 & 2.012 \\
\hline 42 & 0.955 & 0.875 & 0.960 & 0.994 & 0.925 & 0.931 & 4.976 & 4.209 & 6.298 & 7.813 & 4.318 & 4.439 \\
\hline 43 & 0.967 & 0.909 & 0.980 & 0.863 & 0.937 & 0.960 & 0.773 & 0.625 & 0.972 & 1.012 & 0.659 & 0.743 \\
\hline 44 & 0.960 & 0.896 & 0.970 & 0.993 & 0.928 & 0.939 & 2.076 & 1.750 & 2.684 & 3.013 & 1.788 & 1.921 \\
\hline 45 & 0.952 & 0.835 & 0.970 & 0.996 & 0.908 & 0.914 & 4.683 & 3.786 & 7.007 & 7.008 & 3.952 & 4.182 \\
\hline
\end{tabular}

Notes: FG, fiducial generalized confidence interval; $\mathrm{MO}$, method of variance estimates

HJ, HPD-based Jeffreys' rule prior, HPD-JR; HN, HPD-based normal-gamma-beta prior.

Bold denoted as the best-performing method each case. 


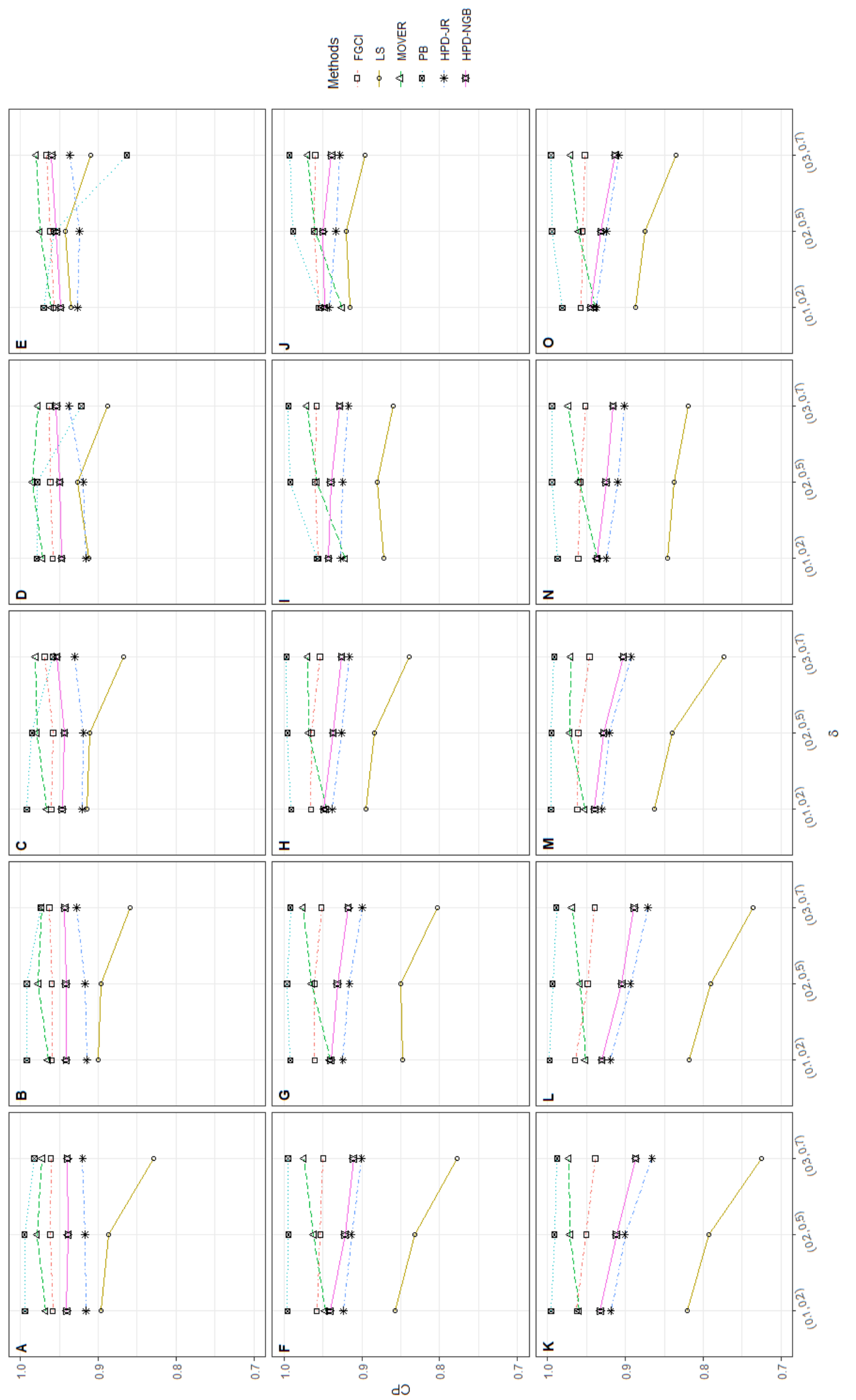

อิ

ति

n'

กิ

¿

ชิ

f8

i

on

อิత

ชิ

in

กิอ

ธิं

(2)

\&

$\ddot{\overrightarrow{0}}$

迆

. త్రి

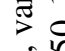

की

w

을

氙

过

है

宇

ह

응 용

巳

$\exists$

ปิ

च

융

芯 0

$\therefore \stackrel{n}{n}$

$\ddot{\circ}$

कृ ?

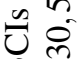

\&

次

近

के

¿ $त$

콩

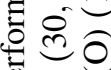

ฉิ્થિ

ชิ

$\therefore$ -

용

운으 
Table 3. Performance measures of 95\%CIs for $\vartheta: 5$ sample cases.

\begin{tabular}{|c|c|c|c|c|c|c|c|c|c|c|c|c|}
\hline \multirow{2}{*}{ Scenarios } & \multicolumn{6}{|l|}{$\mathrm{CP}$} & \multicolumn{6}{|l|}{$\mathrm{AL}$} \\
\hline & FG & LS & MO & $\mathrm{PB}$ & HJ & $\mathrm{HN}$ & FG & LS & MO & $\mathrm{PB}$ & HJ & $\mathrm{HN}$ \\
\hline \multicolumn{13}{|l|}{$k=5$} \\
\hline 46 & 0.885 & 0.790 & 0.988 & 0.989 & 0.757 & 0.846 & 0.963 & 0.819 & 1.794 & 1.532 & 0.848 & 0.956 \\
\hline 47 & 0.789 & 0.627 & 0.973 & 0.996 & 0.674 & 0.715 & 2.240 & 1.908 & 4.982 & 3.897 & 1.991 & 2.176 \\
\hline 48 & 0.840 & 0.613 & 0.953 & 0.997 & 0.723 & 0.746 & 5.325 & 4.529 & 13.769 & 12.250 & 4.744 & 4.870 \\
\hline 49 & 0.894 & 0.800 & 0.993 & 0.978 & 0.779 & 0.864 & 0.900 & 0.765 & 1.825 & 1.439 & 0.773 & 0.905 \\
\hline 50 & 0.783 & 0.623 & 0.972 & 0.998 & 0.680 & 0.711 & 2.008 & 1.711 & 5.203 & 3.608 & 1.750 & 1.955 \\
\hline 51 & 0.797 & 0.580 & 0.959 & 0.996 & 0.680 & 0.701 & 4.700 & 4.066 & 16.626 & 11.353 & 4.118 & 4.287 \\
\hline 52 & 0.893 & 0.735 & 0.989 & 0.896 & 0.816 & 0.853 & 0.753 & 0.589 & 2.849 & 1.433 & 0.636 & 0.764 \\
\hline 53 & 0.768 & 0.517 & 0.977 & 0.997 & 0.666 & 0.676 & 1.474 & 1.168 & 19.967 & 3.364 & 1.282 & 1.406 \\
\hline 54 & 0.742 & 0.467 & 0.983 & 0.996 & 0.624 & 0.629 & 3.250 & 2.654 & $1.5 \mathrm{e} 4$ & 11.238 & 2.817 & 2.855 \\
\hline 55 & 0.884 & 0.779 & 0.988 & 0.979 & 0.743 & 0.846 & 0.940 & 0.777 & 1.739 & 1.434 & 0.857 & 0.930 \\
\hline 56 & 0.806 & 0.645 & 0.973 & 0.995 & 0.681 & 0.740 & 2.204 & 1.822 & 4.586 & 3.561 & 2.045 & 2.141 \\
\hline 57 & 0.858 & 0.622 & 0.949 & 0.986 & 0.725 & 0.771 & 5.620 & 4.542 & 12.575 & 12.073 & 5.122 & 5.162 \\
\hline 58 & 0.901 & 0.827 & 0.995 & 0.962 & 0.770 & 0.870 & 0.845 & 0.728 & 1.699 & 1.326 & 0.771 & 0.841 \\
\hline 59 & 0.793 & 0.644 & 0.978 & 0.997 & 0.675 & 0.726 & 1.904 & 1.629 & 4.351 & 3.262 & 1.750 & 1.850 \\
\hline 60 & 0.825 & 0.605 & 0.952 & 0.997 & 0.710 & 0.734 & 4.753 & 4.058 & 12.745 & 10.793 & 4.373 & 4.353 \\
\hline 61 & 0.905 & 0.785 & 0.992 & 0.822 & 0.809 & 0.865 & 0.685 & 0.564 & 1.632 & 1.219 & 0.620 & 0.686 \\
\hline 62 & 0.786 & 0.578 & 0.969 & 0.993 & 0.683 & 0.704 & 1.368 & 1.142 & 4.477 & 2.775 & 1.260 & 1.309 \\
\hline 63 & 0.755 & 0.496 & 0.963 & 0.998 & 0.639 & 0.637 & 3.177 & 2.714 & 18.995 & 8.911 & 2.884 & 2.822 \\
\hline 64 & 0.892 & 0.787 & 0.991 & 0.970 & 0.737 & 0.858 & 0.928 & 0.751 & 1.740 & 1.364 & 0.872 & 0.919 \\
\hline 65 & 0.822 & 0.647 & 0.975 & 0.996 & 0.673 & 0.763 & 2.168 & 1.738 & 4.371 & 3.326 & 2.047 & 2.114 \\
\hline 66 & 0.852 & 0.593 & 0.943 & 0.981 & 0.715 & 0.767 & 5.710 & 4.413 & 12.195 & 11.422 & 5.267 & 5.278 \\
\hline 67 & 0.905 & 0.827 & 0.996 & 0.949 & 0.768 & 0.873 & 0.816 & 0.697 & 1.637 & 1.256 & 0.770 & 0.811 \\
\hline 68 & 0.801 & 0.654 & 0.979 & 0.995 & 0.683 & 0.737 & 1.839 & 1.549 & 4.069 & 3.016 & 1.753 & 1.797 \\
\hline 69 & 0.821 & 0.595 & 0.947 & 0.994 & 0.693 & 0.733 & 4.806 & 3.976 & 12.174 & 10.326 & 4.431 & 4.432 \\
\hline 70 & 0.917 & 0.803 & 0.994 & 0.775 & 0.817 & 0.886 & 0.650 & 0.539 & 1.499 & 1.133 & 0.616 & 0.650 \\
\hline 71 & 0.804 & 0.612 & 0.973 & 0.992 & 0.692 & 0.730 & 1.310 & 1.094 & 3.962 & 2.543 & 1.236 & 1.262 \\
\hline 72 & 0.756 & 0.502 & 0.958 & 0.997 & 0.631 & 0.646 & 3.158 & 2.695 & 16.604 & 8.356 & 2.888 & 2.835 \\
\hline 73 & 0.924 & 0.832 & 0.994 & 0.942 & 0.772 & 0.893 & 0.822 & 0.673 & 1.505 & 1.186 & 0.856 & 0.808 \\
\hline 74 & 0.853 & 0.699 & 0.985 & 0.990 & 0.696 & 0.798 & 1.971 & 1.589 & 3.8 & 2.899 & 2.000 & 1.923 \\
\hline 75 & 0.883 & 0.652 & 0.952 & 0.945 & 0.755 & 0.817 & 5.330 & 4.072 & 9.997 & 9.911 & 5.224 & 4.974 \\
\hline 76 & 0.924 & 0.857 & 0.997 & 0.913 & 0.771 & 0.901 & 0.723 & 0.626 & 1.418 & 1.088 & 0.746 & 0.715 \\
\hline 77 & 0.826 & 0.695 & 0.986 & 0.989 & 0.689 & 0.767 & 1.670 & 1.4 & 76 & 610 & 992 & 1.632 \\
\hline 78 & 0.854 & 0.638 & 0.955 & 0.984 & 0.718 & 0.771 & 4.456 & 3.628 & 9.715 & 788 & 4.311 & 4.160 \\
\hline 79 & 0.930 & 0.846 & 0.998 & 0.683 & 0.811 & 0.900 & 0.581 & 0.486 & 1.253 & 0.964 & 0.586 & 0.580 \\
\hline 80 & 0.830 & 0.658 & 0.981 & 0.980 & 0.705 & 0.762 & 1.215 & 1.019 & 3.179 & 2.168 & 1.225 & 1.181 \\
\hline 81 & 0.788 & 0.555 & 0.967 & 0.997 & 0.675 & 0.689 & 2.992 & 2.554 & 11.873 & 7.026 & 2.927 & 2.738 \\
\hline 82 & 0.915 & 0.844 & 0.993 & 0.964 & 0.788 & 0.889 & 0.769 & 0.662 & 1.3 & 1.158 & 0.692 & 0.753 \\
\hline 83 & 0.858 & 0.735 & 0.982 & 0.993 & 0.741 & 0.804 & 1.882 & 1.599 & 3.605 & 2.920 & 1.698 & 1.825 \\
\hline 84 & 0.886 & 0.705 & 0.969 & 0.981 & 0.782 & 0.827 & 4.650 & 3.895 & 8.767 & 9.068 & 4.208 & 4.335 \\
\hline 85 & 0.925 & 0.865 & 0.998 & 0.939 & 0.803 & 0.897 & 0.707 & 0.618 & 1.315 & 1.068 & 0.618 & 0.700 \\
\hline 86 & 0.834 & 0.705 & 0.987 & 0.994 & 0.735 & 0.775 & 1.683 & 1.439 & 3.493 & 2.683 & 1.482 & 1.642 \\
\hline 87 & 0.855 & 0.684 & 0.968 & 0.994 & 0.751 & 0.783 & 4.027 & 3.489 & 8.924 & 8.068 & 3.613 & 3.766 \\
\hline 88 & 0.929 & 0.824 & 0.994 & 0.677 & 0.835 & 0.903 & 0.611 & 0.495 & 1.322 & 0.993 & 0.515 & 0.616 \\
\hline 89 & 0.823 & 0.627 & 0.981 & 0.985 & 0.729 & 0.749 & 1.284 & 1.045 & 3.692 & 2.296 & 1.121 & 1.250 \\
\hline 90 & 0.799 & 0.578 & 0.972 & 0.997 & 0.699 & 0.705 & 2.875 & 2.453 & 13.603 & 6.644 & 2.519 & 2.641 \\
\hline 91 & 0.927 & 0.831 & 0.997 & 0.906 & 0.777 & 0.898 & 0.753 & 0.614 & 1.389 & 1.064 & 0.703 & 0.735 \\
\hline 92 & 0.871 & 0.731 & 0.988 & 0.986 & 0.720 & 0.820 & 1.821 & 1.466 & 3.459 & 2.601 & 1.721 & 1.769 \\
\hline 93 & 0.905 & 0.693 & 0.957 & 0.897 & 0.791 & 0.852 & 5.015 & 3.768 & 8.461 & 8.829 & 4.621 & 4.690 \\
\hline 94 & 0.931 & 0.879 & 0.999 & 0.873 & 0.781 & 0.909 & 0.651 & 0.571 & 1.279 & 0.972 & 0.608 & 0.639 \\
\hline 95 & 0.847 & 0.738 & 0.991 & 0.986 & 0.719 & 0.797 & 1.541 & 1.313 & 3.117 & 2.351 & 1.447 & 1.499 \\
\hline 96 & 0.875 & 0.679 & 0.966 & 0.969 & 0.760 & 0.806 & 4.125 & 3.374 & 8.002 & 7.707 & 3.808 & 3.865 \\
\hline 97 & 0.935 & 0.866 & 0.998 & 0.541 & 0.832 & 0.911 & 0.529 & 0.450 & 1.097 & 0.856 & 0.493 & 0.523 \\
\hline 98 & 0.848 & 0.697 & 0.986 & 0.971 & 0.735 & 0.782 & 1.126 & 0.956 & 2.572 & 1.916 & 1.060 & 1.091 \\
\hline 99 & 0.817 & 0.613 & 0.963 & 0.994 & 0.698 & 0.725 & 2.784 & 2.418 & 7.510 & 6.042 & 2.565 & 2.571 \\
\hline 100 & 0.941 & 0.888 & 0.998 & 0.863 & 0.813 & 0.920 & 0.557 & 0.484 & 0.954 & 0.806 & 0.510 & 0.536 \\
\hline 101 & 0.906 & 0.827 & 0.995 & 0.973 & 0.799 & 0.875 & 1.413 & 1.201 & 2.515 & 2.029 & 1.288 & 1.361 \\
\hline 102 & 0.929 & 0.790 & 0.975 & 0.861 & 0.845 & 0.889 & 3.639 & 2.946 & 5.529 & 6.174 & 3.365 & 3.428 \\
\hline 103 & 0.948 & 0.923 & 1.000 & 0.801 & 0.816 & 0.931 & 0.501 & 0.456 & 0.909 & 0.741 & 0.452 & 0.487 \\
\hline 104 & 0.888 & 0.816 & 0.996 & 0.978 & 0.784 & 0.853 & 1.253 & 1.095 & 2.373 & 1.852 & 1.121 & 1.216 \\
\hline 105 & 0.905 & 0.775 & 0.981 & 0.953 & 0.822 & 0.859 & 3.147 & 2.678 & 5.326 & 5.441 & 2.893 & 2.975 \\
\hline 106 & 0.955 & 0.907 & 0.999 & 0.289 & 0.852 & 0.943 & 0.438 & 0.372 & 0.838 & 0.668 & 0.373 & 0.433 \\
\hline 107 & 0.881 & 0.761 & 0.994 & 0.939 & 0.781 & 0.833 & 0.992 & 0.823 & 2.044 & 1.536 & 0.863 & 0.972 \\
\hline 108 & 0.868 & 0.722 & 0.984 & 0.987 & 0.781 & 0.805 & 2.331 & 2.005 & 5.072 & 4.308 & 2.088 & 2.208 \\
\hline
\end{tabular}

Notes: FG, fiducial generalized confidence interval; MO, method of variance estimates recovery;

HJ, HPD-based Jeffreys' rule prior, HPD-JR; HN, HPD-based normal-gamma-beta prior.

Bold denoted as the best-performing method each case. 


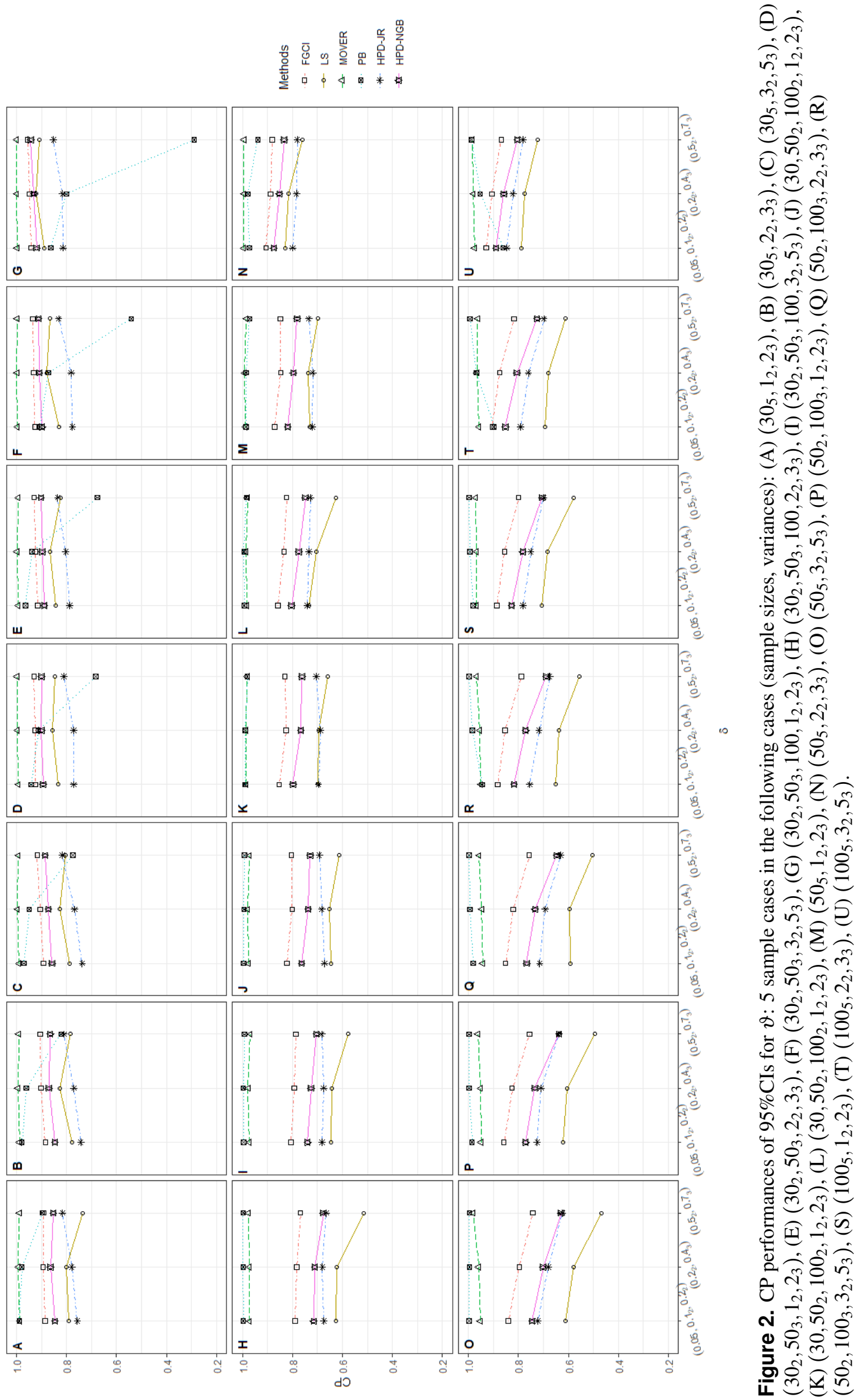


Table 4. Performance measures of $95 \%$ CIs for $\vartheta: 10$ sample cases.

\begin{tabular}{|c|c|c|c|c|c|c|c|c|c|c|c|c|}
\hline \multirow{2}{*}{ Scenarios } & \multicolumn{6}{|l|}{$\mathrm{CP}$} & \multicolumn{6}{|l|}{$\mathrm{AL}$} \\
\hline & FG & LS & MO & PB & HJ & HN & $\overline{\text { FG }}$ & LS & MO & PB & HJ & HN \\
\hline \multicolumn{13}{|l|}{$k=10$} \\
\hline 109 & 0.728 & 0.675 & 0.998 & 0.927 & 0.566 & 0.692 & 0.612 & 0.501 & 1.554 & 0.932 & 0.545 & 0.623 \\
\hline 110 & 0.661 & 0.500 & 0.979 & 0.891 & 0.570 & 0.588 & 1.644 & 1.291 & 3.867 & 3.278 & 1.500 & 1.637 \\
\hline 111 & 0.504 & 0.352 & 0.950 & 0.978 & 0.481 & 0.404 & 3.159 & 2.561 & 8.645 & 7.286 & 2.996 & 3.076 \\
\hline 112 & 0.720 & 0.692 & 0.999 & 0.904 & 0.587 & 0.690 & 0.557 & 0.459 & 1.519 & 0.832 & 0.483 & 0.574 \\
\hline 113 & 0.532 & 0.452 & 0.976 & 0.985 & 0.512 & 0.462 & 1.393 & 1.159 & 3.853 & 2.682 & 1.260 & 1.404 \\
\hline 114 & 0.361 & 0.290 & 0.955 & 0.998 & 0.403 & 0.274 & 2.556 & 2.218 & 8.570 & 5.943 & 2.411 & 2.505 \\
\hline 115 & 0.789 & 0.723 & 0.999 & 0.808 & 0.561 & 0.762 & 0.554 & 0.440 & 1.416 & 0.789 & 0.546 & 0.560 \\
\hline 116 & 0.716 & 0.524 & 0.985 & 0.578 & 0.590 & 0.653 & 1.635 & 1.180 & 3.478 & 2.915 & 1.559 & 1.624 \\
\hline 117 & 0.593 & 0.406 & 0.964 & 0.872 & 0.519 & 0.507 & 3.289 & 2.406 & 7.754 & 6.380 & 3.189 & 3.214 \\
\hline 118 & 0.782 & 0.773 & 1.000 & 0.780 & 0.586 & 0.758 & 0.477 & 0.404 & 1.317 & 0.696 & 0.474 & 0.483 \\
\hline 119 & 0.626 & 0.514 & 0.988 & 0.947 & 0.535 & 0.561 & 1.337 & 1.076 & 3.348 & 2.360 & 1.284 & 1.341 \\
\hline 120 & 0.447 & 0.347 & 0.965 & 0.992 & 0.450 & 0.355 & 2.570 & 2.108 & 7.290 & 5.180 & 2.506 & 2.531 \\
\hline 121 & 0.826 & 0.773 & 1.000 & 0.736 & 0.592 & 0.796 & 0.488 & 0.399 & 1.266 & 0.695 & 0.444 & 0.486 \\
\hline 122 & 0.774 & 0.620 & 0.994 & 0.438 & 0.647 & 0.720 & 1.460 & 1.086 & 3.072 & 2.512 & 1.328 & 1.438 \\
\hline 123 & 0.659 & 0.460 & 0.977 & 0.798 & 0.553 & 0.577 & 3.002 & 2.236 & 6.597 & 5.502 & 2.775 & 2.921 \\
\hline 124 & 0.828 & 0.826 & 1.000 & 0.708 & 0.606 & 0.802 & 0.426 & 0.368 & 1.187 & 0.615 & 0.387 & 0.427 \\
\hline 125 & 0.688 & 0.595 & 0.995 & 0.912 & 0.591 & 0.627 & 1.205 & 0.992 & 2.912 & 2.039 & 1.094 & 1.197 \\
\hline 126 & 0.520 & 0.426 & 0.979 & 0.984 & 0.486 & 0.439 & 2.390 & 1.989 & 6.222 & 4.479 & 2.224 & 2.344 \\
\hline
\end{tabular}

Notes: FG, fiducial generalized confidence interval; MO, method of variance estimates recovery;

HJ, HPD-based Jeffreys' rule prior, HPD-JR; HN, HPD-based normal-gamma-beta prior.

Bold denoted as the best-performing method each case.

\section{AN EMPIRICAL APPLICATION}

Daily rainfall data obtained from the Thai Meteorological Department (TMD) were divided into the northern, northeastern, central, and eastern regions, while the southern region was a combination of the data from the southeastern and southwestern shores. Due to the differences in the climate patterns and meteorological conditions in the five regions, we focused was on estimating the daily rainfall data in these regions by treating them as separate sets of observations rather than using the average rainfall for the whole of Thailand by pooling them and treating them as a single population. The daily rainfall amounts were recorded on August 5 and 9,2019, which is in the middle of the rainy season (mid-May to mid-October) when rice farming is conducted in Thailand. Entries with rainfall of less than $0.1 \mathrm{~mm}$ were considered as zero records.

Tables 5-6 contain the daily rainfall records for the five regions, while Figures 4-5 show histogram plots of rainfall observations, and Figures 6-7 exhibit normal Q-Q plots of the log-positive rainfall data on August 5 and 9, 2019, respectively. It can be seen that the data for all of the regions contained zero observations. After that, the fitted distribution of the positive observations was checked using the Akaike information criterion (AIC), as reported in Table 7. It can be concluded that the rainfall data in all of the regions on August 5 and 9, 2019 follow a delta-lognormal distribution. All data sets and R code are available in the Supplemental Files. The summary statistics are reported in Table 8. In the approximation of the daily rainfall amounts in the five regions, the estimated common means were 4.4506 and 13.2621 $\mathrm{mm} /$ day on August 5 and 9, 2019, respectively. The computed 95\%CIs of the common rainfall mean are reported in Table 9. Under the rain criteria issued by the TMD (Department, 2018), it can be interpreted that the daily rainfall in Thailand on August 5, 2019, was light $(0.1-10.0 \mathrm{~mm})$, while it was moderate (10.1-35.0 mm) on August 9, 2019. These results confirm the simulation results for $k=5$ in the previous section.

\section{DISCUSSION}

It can be seen that for MOVER and PB developed from the studies of Krishnamoorthy and Oral (2015) and Malekzadeh and Kharrati-Kopaei (2019), respectively, the simulation results are similar to both of these studies provided that the zero observations are omitted. CIs for the common mean have been investigated in both normal and lognormal distributions (Fairweather, 1972; Jordan and Krishnamoorthy, 1996; Krishnamoorthy and Mathew, 2003; Lin and Lee, 2005; Tian and Wu, 2007; Krishnamoorthy and Oral, 2015). However, the common mean of delta-lognormal populations is especially of interest because 
Table 5. Daily rainfall data in five Thailand's regions on August 5, 2019.

\begin{tabular}{|c|c|c|c|c|c|c|c|c|c|c|c|c|c|c|c|}
\hline \multicolumn{2}{|c|}{ Northern } & \multicolumn{7}{|c|}{ Northeastern } & \multicolumn{2}{|c|}{ Central } & \multirow{2}{*}{$\begin{array}{r}\text { Eastern } \\
0\end{array}$} & \multicolumn{4}{|c|}{ Southern } \\
\hline 3 & 0 & 3 & 0 & 0 & 49.5 & 0 & 0 & 0 & 2.9 & 3.2 & & 4.1 & 0 & 0 & 2.7 \\
\hline 2.6 & 5 & 0 & 40 & 1.5 & 10.5 & 0 & 0 & 0 & 0.2 & 0 & 3.2 & 0 & 0 & 0 & 0 \\
\hline 1 & 23.8 & 0 & 3.5 & 18.5 & 60.4 & 4 & 0 & 11 & 0.3 & 0 & 10.4 & 11.5 & 3.5 & 0 & 0 \\
\hline 3.6 & 16 & 0 & 0 & 42 & 12.7 & 0 & 0 & 0 & 2.5 & 4.7 & 1.1 & 2.5 & 13.6 & 0 & 0 \\
\hline 0 & 11.5 & 0 & 12 & 9.1 & 6.8 & 0 & 20.3 & 0 & 0.4 & 19.3 & 0.2 & 9.7 & 0 & 0.2 & 0 \\
\hline 13.2 & 1.2 & 0 & 15 & 6 & 69.3 & 0 & 0 & 0 & 0.4 & 3.1 & 4.3 & 10.4 & 0 & 0 & 0 \\
\hline 22.4 & 10.3 & 0 & 0 & 7.5 & 36.5 & 0 & 2.4 & 0.3 & 1.1 & 2.9 & 0 & 9.6 & 0 & 0 & 0 \\
\hline 1.4 & 1.7 & 0 & 1.5 & 0 & 8.6 & 0 & 0 & 1 & 0 & 5.7 & 0 & 19 & 0 & 0 & 0 \\
\hline 18.3 & 5.5 & 0 & 0.7 & 6.3 & 0 & 0 & 0 & 0 & 1.3 & 0.9 & 0 & 8.3 & 0 & 0 & 0 \\
\hline 0 & 7.3 & 0 & 0 & 0 & 0 & 0 & 0 & 0 & 0.1 & 0 & 0 & 0 & 4.8 & 0 & 6.2 \\
\hline 15.5 & 24.3 & 1.7 & 3 & 0.4 & 0 & 0 & 0 & 0 & 2.9 & 0 & 0.2 & 0 & 0 & 0 & 0 \\
\hline 0 & 27.2 & 2.3 & 0 & 0 & 3.8 & 0 & 0 & 0 & 0 & 2.6 & 0.1 & 0 & 0 & 0 & 0 \\
\hline 0 & 12.6 & 0.5 & 0 & 0 & 0 & 0 & 3.2 & 0 & 1 & 17 & 62.8 & 0 & 0 & 0 & 6.1 \\
\hline 0 & 22.7 & 3.9 & 0 & 0 & 0 & 0 & 0 & 0 & 4.7 & 0 & 36.7 & 17.8 & 0 & 0 & 0 \\
\hline 9.8 & 0 & 6.9 & 29.4 & 1.8 & 0 & 0 & 0 & 0 & 0.5 & 3.5 & 15.6 & 12.3 & 0 & 0 & 0 \\
\hline 24.3 & 2.6 & 2.2 & 48 & 0 & 0 & 0 & 0 & 0 & 5 & 0 & 50 & 2.5 & 0 & 0 & 0 \\
\hline 24.6 & 0 & 3.2 & 0 & 0 & 0 & 6 & 0 & 0 & 2.5 & 0 & 35.5 & 0 & 0 & 0 & 0.3 \\
\hline 8.8 & 3.2 & 5.3 & 70.8 & 14.3 & 0 & 0 & 0 & 0 & 0 & 0 & 35 & 0.9 & 0 & 0 & 0 \\
\hline 0 & 2.6 & 11 & 3.5 & 0 & 0 & 0 & 0 & 0 & 0 & 5.1 & 5.9 & 0 & 0 & 0 & 0 \\
\hline 19.8 & 2 & 0.6 & 14.2 & 0 & 0 & 0 & 4.8 & 0 & 0 & 60.4 & 0 & 2.6 & 0 & 0 & 0 \\
\hline 5 & 8 & 0 & 7 & 0 & 0 & 2.3 & 0 & 0 & 0 & 6.9 & 0 & 0 & 0 & 0 & 0 \\
\hline 12.3 & 1.9 & 1 & 0 & 0 & 21.5 & 0 & 0 & 0 & 6.6 & 3 & 3 & 0 & 0 & 0 & 0 \\
\hline 8.1 & 0.8 & 2.4 & 0 & 0 & 2.5 & 1 & 0 & 0 & 0 & 15.1 & 60.4 & 2 & 0 & 0 & 0 \\
\hline 4.8 & 2.2 & 13.2 & 0 & 0 & 0 & 0 & 0 & 0 & 9.5 & 6 & 60 & 0 & 0 & 0 & 0 \\
\hline 5.8 & 6.5 & 0.4 & 0 & 0 & 13 & 0 & 0 & & 5.1 & 13.4 & 76 & 0 & 0 & 0 & 0 \\
\hline 17 & 0 & 0 & 10.8 & 0 & 26.2 & 0 & 0 & & 12.5 & 6.2 & 79.7 & 0 & 0 & 0 & 0 \\
\hline 25.1 & 2.2 & 1.3 & 0 & 10.1 & 2.2 & 4.6 & 5.4 & & 0 & & 65.7 & 3.5 & 0 & 0 & \\
\hline 8.3 & 0 & 10 & 6.3 & 0 & 3 & 0 & 0 & & 0 & & 108 & 0 & 0 & 36.1 & \\
\hline 22.9 & 4.3 & 2.5 & 0 & 4.8 & 10.5 & 10 & 0 & & 3.2 & & 10.5 & 0 & 0 & 41.8 & \\
\hline 26.9 & 0.2 & 4.6 & 4 & 0 & 0 & 0 & 12 & & 0 & & & 0 & 0 & 30 & \\
\hline 0 & 0 & 0 & 19.3 & 0 & 0 & 9.5 & 0 & & 2.2 & & & 0 & 0 & 0 & \\
\hline
\end{tabular}

Source: Thai Meteorological Department

https://www.tmd.go.th/services/weekly_report.php

it can be used to fit the data from real-world situations such as investigating medical costs (Zou et al., 2009; Tierney et al., 2003; Tian, 2005), analyzing airborne contaminants (Owen and DeRouen, 1980; Tian, 2005) and measuring fish abundance (Fletcher, 2008; Wu and Hsieh, 2014). Furthermore, it is possible that some extreme rainfall data also fulfill the assumptions of a delta-lognormal distribution. Note that such natural disasters as floods and landslides have been caused by the extreme rainfall events, as evidenced in many country around the world: Europe (e.g. Northern England, Southern Scotland and Ireland (Otto and Oldenborgh, 2017)), Asia (e.g. Japan (Oldenborgh, 2018)) and North America (e.g. Southeast Texas (Oldenborgh et al., 2019)). Our findings show that some of the methods studied had CPs that were too low or too high for large sample cases, a shortcoming that should be addressed in future work.

\section{CONCLUSIONS}

The objective of this study was to propose CIs for the common mean of several delta-lognormal distributions using FGCI, LS, MOVER, PB, HPD-JR, and HPD-NGB. The CP and AL as performance measures of the methods were assessed via Monte Carlo simulation. The findings confirm that for small sample case $(k=2)$, FGCI and HPD-NGB are the recommended methods in different situations: FGCI (a small-to-moderate sample size and a large $\sigma_{i}^{2}$ with a moderate-to-large sample size) and HPD-NGB (small $\sigma_{i}^{2}$ with a moderate-to-large sample size). For large sample cases $(k=5,10)$, MOVER ( and $\sigma_{i}^{2}$ ) and PB (large $\delta_{i}$ and $\sigma_{i}^{2}$ ) performed the best.

\section{ACKNOWLEDGMENTS}

The authors are grateful to the academic editor and reviewers for their constructive comments and suggestions which help to improve this manuscript. 
Table 6. Daily rainfall data in five Thailand's regions on August 9, 2019.

\begin{tabular}{|c|c|c|c|c|c|c|c|c|c|c|c|c|c|c|c|}
\hline \multicolumn{2}{|c|}{ Northern } & \multicolumn{7}{|c|}{ Northeastern } & \multicolumn{2}{|c|}{ Central } & \multirow{2}{*}{$\begin{array}{r}\text { Eastern } \\
0\end{array}$} & \multicolumn{4}{|c|}{ Southern } \\
\hline 9.5 & 0 & 25.3 & 20 & 6.6 & 8.4 & 0 & 67 & 0 & 39.6 & 0 & & 27.9 & 4.1 & 0.4 & 114.6 \\
\hline 4.9 & 10 & 25.5 & 14.5 & 16.9 & 0.8 & 2.9 & 65.4 & 0 & 25 & 0 & 0 & 0 & 9 & 3.8 & 0 \\
\hline 0 & 21.6 & 24 & 3 & 10 & 20.2 & 0 & 21 & 0 & 0 & 0 & 26.5 & 3.4 & 27.3 & 0.6 & 0 \\
\hline 4.7 & 15 & 8 & 28 & 48.2 & 0 & 14.3 & 6.4 & 7.2 & 0 & 0 & 36.4 & 0 & 6.5 & 0 & 0 \\
\hline 0 & 15.5 & 0 & 27 & 6.5 & 0.5 & 0 & 0 & 3.5 & 29.7 & 0.1 & 0 & 0.8 & 3.5 & 10.8 & 0 \\
\hline 63.2 & 14 & 20 & 50 & 4.8 & 5.3 & 6 & 52 & 0 & 0 & 0.3 & 4.5 & 37.9 & 0 & 5 & 18.2 \\
\hline 9.6 & 8.5 & 0 & 24 & 25 & 16.7 & 0 & 45 & 40.5 & 3.1 & 0.5 & 0 & 32.4 & 0 & 12.2 & 40.4 \\
\hline 10.7 & 11.5 & 0 & 30 & 0 & 45.2 & 28 & 41.4 & 25.8 & 8.2 & 31.5 & 0.5 & 33.8 & 0 & 3.6 & 0 \\
\hline 13 & 17.4 & 0 & 22 & 0 & 0 & 0 & 14.3 & 30.4 & 3.2 & 8.2 & 0.7 & 15.8 & 0 & 0 & 0 \\
\hline 0 & 15.6 & 0 & 16 & 3.2 & 0.6 & 0 & 45 & 0 & 7.1 & 0 & 12.3 & 0 & 3.6 & 8.8 & 10.8 \\
\hline 0 & 31.6 & 33.8 & 0 & 44 & 0 & 0 & 27 & 0 & 0 & 0 & 0.5 & 0 & 3 & 0 & 0 \\
\hline 0 & 20.6 & 33.7 & 0 & 0 & 3.1 & 27.6 & 0.2 & 0 & 3.2 & 0 & 1.9 & 0 & 1 & 0 & 0 \\
\hline 0 & 31.1 & 15.1 & 0 & 9.3 & 33.3 & 33 & 30 & 0 & 4.2 & 0 & 66.4 & 0 & 3.7 & 6.2 & 35 \\
\hline 0 & 16.3 & 18.5 & 0 & 0 & 6 & 0 & 0 & 0 & 5.7 & 0 & 93.6 & 11.5 & 15.6 & 0 & 0 \\
\hline 2.8 & 0 & 44.8 & 39.7 & 20 & 0 & 0 & 0 & 8.3 & 30 & 0 & 68.7 & 1.7 & 11.2 & 3.8 & 33.5 \\
\hline 11.3 & 33.1 & 37.5 & 9.3 & 0 & 13.2 & 0 & 0 & 0 & 4 & 0 & 40 & 1.2 & 24 & 0 & 57 \\
\hline 0.6 & 29.2 & 0 & 0 & 4.8 & 0 & 0 & 0 & 0 & 0 & 0 & 65 & 21.2 & 0 & 0 & 10.5 \\
\hline 36.1 & 11.2 & 47 & 2.1 & 0 & 21 & 0 & 0 & 0 & 0 & 0 & 63.7 & 0 & 0 & 0 & 0 \\
\hline 0 & 14.4 & 20 & 0 & 0 & 0 & 0 & 1 & 36.1 & 0 & 0 & 9.2 & 30 & 10.2 & 0.2 & 0 \\
\hline 2.6 & 60 & 30.8 & 46.7 & 0 & 8.4 & 15 & 0 & 0 & 0 & 1.2 & 0 & 5.1 & 0 & 0 & 0 \\
\hline 5 & 42.3 & 30 & 10.5 & 0 & 0 & 0 & 0 & 12.5 & 0 & 0 & 0 & 2.5 & 0 & 0 & 30.8 \\
\hline 13.4 & 9.5 & 1 & 0 & 56.5 & 0 & 0 & 2.5 & 0 & 14.7 & 0.1 & 11 & 2.4 & 0 & 0.4 & 10.7 \\
\hline 12.3 & 34.5 & 1.2 & 41 & 39.2 & 0.5 & 0 & 0 & 0 & 0 & 1 & 69.6 & 5 & 0 & 0 & 0 \\
\hline 25.8 & 36.5 & 56.3 & 10.3 & 0 & 4.5 & 25.7 & 9.5 & 0 & 0 & 3 & 89.6 & 1.7 & 0 & 0 & 15.9 \\
\hline 30.2 & 9.7 & 0 & 1.2 & 6.4 & 16.2 & 41.4 & 0 & & 0 & 0.5 & 160 & 0 & 0 & 2.2 & 0 \\
\hline 16.4 & 0 & 6 & 23.9 & 5.3 & 0 & 41.6 & 0 & & 0 & 1.6 & 34.3 & 0 & 0 & 0 & 0 \\
\hline 6 & 0 & 0 & 22.2 & 0 & 3.5 & 53.8 & 0 & & 0 & & 0 & 2.1 & 0 & 0.6 & \\
\hline 33.1 & 7.6 & 5.3 & 24.1 & 9.8 & 20 & 48.5 & 0 & & 0 & & 25 & 0 & 0 & 76.6 & \\
\hline 16.4 & 9.6 & 7.2 & 38 & 0 & 0 & 78.5 & 2.1 & & 0 & & 19.5 & 10.5 & 7 & 121.6 & \\
\hline 19.8 & 9.3 & 24.6 & 9 & 9.7 & 0 & 12.7 & 0 & & 0 & & & 15.3 & 10.6 & 60 & \\
\hline 0 & 0 & 30 & 9.2 & 4.5 & 1.2 & 80.9 & 0 & & 0 & & & 0 & 3.6 & 0 & \\
\hline
\end{tabular}

Source: Thai Meteorological Department

URL: https://www.tmd.go.th/services/weekly_report.php

\section{REFERENCES}

Aitchison, J. (1955). On the distribution of a positive random variable having a discrete probability mass at the origin. Journal of the American Statistical Association, 50(271):901-908.

Aitchison, J. and Brown, J. A. (1963). The lognormal distribution: with special reference to its uses in economics. Cambridge University Press.

Box, G. E. P. and Tiao, G. C. (1973). Bayesian Inference in Statistical Analysis. New York: Wiley Classics.

Casella, G. and Berger, R. L. (2002). Statistical Inference (2nd ed.). Duxbury, Pacifc Grove, CA.

Department, T. M. (2018). Meteorological academic, knowledge and document. Available from https://www.tmd.go.th/info/info.php?FileID=68.

Fairweather, W. R. (1972). A method of obtaining an exact confidence interval for the common mean of several normal populations. Journal of the Royal Statistical Society. Series C (Applied Statistics), 21(3):229-233.

Fisher, R. A. (1930). Inverse Probability. Mathematical Proceedings of the Cambridge Philosophical Society, 26(4):528-535.

Fletcher, D. (2008). Confidence intervals for the mean of the delta-lognormal distribution. Environmental and Ecological Statistics, 15(2):175-189.

Hannig, J. (2009). On generalized fiducial inference. Statistica Sinica, 19(2):491-544.

Hannig, J., Iyer, H., and Patterson, P. (2006). Fiducial generalized confidence intervals. Journal of the American Statistical Association, 101(473):254-269.

Harvey, J. and van der Merwe, A. J. (2012). Bayesian confidence intervals for means and variances of lognormal and bivariate lognormal distributions. Journal of Statistical Planning and Inference, 142(6):1294-1309.

Hasan, M. S. and Krishnamoorthy, K. (2018). Confidence intervals for the mean and a percentile based on zero-inflated lognormal data. Journal of Statistical Computation and Simulation, 88(8):1499-1514. 
Table 7. AIC results of daily rainfall records in five Thailand's regions.

\begin{tabular}{lrrrrr}
\hline \multirow{2}{*}{ Regions } & \multicolumn{5}{c}{ AIC } \\
\cline { 2 - 6 } & \multicolumn{1}{c}{ Cauchy } & \multicolumn{1}{c}{ Logistic } & Lognormal & Normal & T-distribution \\
\hline On August 5, 2019 & & & & \\
Northern & 373.1958 & 357.3122 & $\mathbf{3 3 6 . 8 7 2 4}$ & 353.7757 & 354.3055 \\
Northeastern & 600.9473 & 642.1779 & $\mathbf{5 4 3 . 9 6 1 9}$ & 667.2334 & 664.6152 \\
Central & 240.0227 & 266.4162 & $\mathbf{2 2 0 . 8 5 0 3}$ & 293.9151 & 283.2302 \\
Eastern & 229.8995 & 220.2523 & $\mathbf{2 0 2 . 8 3 9 4}$ & 218.7240 & 219.1471 \\
Southern & 194.9368 & 197.5586 & $\mathbf{1 7 8 . 5 5 8 7}$ & 201.1654 & 200.1388 \\
\hline On August 9, 2019 & & & & \\
Northern & 389.6257 & 387.3072 & $\mathbf{3 7 5 . 7 9 9 4}$ & 391.1802 & 390.2479 \\
Northeastern & 1123.7491 & 1080.8694 & $\mathbf{1 0 5 2 . 8 9 5 3}$ & 1080.1467 & 1079.9365 \\
Central & 178.8516 & 189.5353 & $\mathbf{1 5 5 . 0 2 6 1}$ & 190.6855 & 190.5103 \\
Eastern & 233.5236 & 227.1725 & $\mathbf{2 1 5 . 9 3 0 6}$ & 228.0501 & 227.4559 \\
Southern & 541.0477 & 569.2615 & $\mathbf{4 8 7 . 4 6 6 7}$ & 592.2242 & 588.2377 \\
\hline
\end{tabular}

Table 8. The summary statistics.

\begin{tabular}{|c|c|c|c|c|c|}
\hline \multirow{2}{*}{ Regions } & \multicolumn{5}{|c|}{ Estimated parameters } \\
\hline & $n_{i}$ & $\hat{\mu}_{i}$ & $\hat{\sigma}_{i}^{2}$ & $\hat{\delta}_{i}$ & $\hat{\vartheta}_{i}$ \\
\hline \multicolumn{6}{|c|}{ August 5, 2020} \\
\hline Northern & 62 & 1.866 & 1.277 & 0.210 & 9.472 \\
\hline Northeastern & 210 & 1.734 & 1.578 & 0.619 & 4.668 \\
\hline Central & 57 & 1.085 & 1.784 & 0.316 & 4.741 \\
\hline Eastern & 29 & 2.366 & 4.545 & 0.241 & 59.391 \\
\hline Southern & 119 & 1.684 & 1.730 & 0.782 & 2.639 \\
\hline \multicolumn{6}{|c|}{ August 9, 2020} \\
\hline Northern & 62 & 2.621 & 0.732 & 0.226 & 15.187 \\
\hline Northeastern & 210 & 2.577 & 1.502 & 0.405 & 16.429 \\
\hline Central & 57 & 1.190 & 3.054 & 0.579 & 5.542 \\
\hline Eastern & 29 & 2.860 & 3.070 & 0.241 & 52.813 \\
\hline Southern & 119 & 2.007 & 2.051 & 0.462 & 10.811 \\
\hline
\end{tabular}

Jordan, S. M. and Krishnamoorthy, K. (1996). Exact confidence intervals for the common mean of several normal populations. Biometrics, 52(1):77-86.

Krishnamoorthy, K. and Mathew, T. (2003). Inferences on the means of lognormal distributions using generalized p-values and generalized confidence intervals. Journal of Statistical Planning and Inference, 115(1):103-121.

Krishnamoorthy, K. and Oral, E. (2015). Standardized likelihood ratio test for comparing several lognormal means and confidence interval for the common mean. Statistical Methods in Medical Research, 26(6):2919-2937.

Kunio, S. (1983). Variances of UMVU Estimators for Means and Variances After Using a Normalizing Transformation. Communications in Statistics - Theory and Methods, 12(9):975-985.

Li, X., Zhou, X., and Tian, L. (2013). Interval estimation for the mean of lognormal data with excess zeros. Statistics \& Probability Letters, 83(11):2447-2453.

Lin, S.-H. and Lee, J. C. (2005). Generalized inferences on the common mean of several normal populations. Journal of Statistical Planning and Inference, 134(2):568-582.

Lin, S. H. and Wang, R. S. (2013). Modified method on the means for several log-normal distributions. Journal of Applied Statistics, 40(1):194-208.

Longford, N. T. (2009). Inference with the lognormal distribution. Journal of Statistical Planning and Inference, 139(7):2329-2340.

Malekzadeh, A. and Kharrati-Kopaei, M. (2019). Inferences on the common mean of several heterogeneous log-normal distributions. Journal of Applied Statistics, 46(6):1066-1083.

Maneerat, P., Niwitpong, S.-a., and Niwitpong, S. (2020). A Bayesian approach to construct confidence intervals for comparing the rainfall dispersion in Thailand. Peerj, 8:e8502.

Miroshnikov, A., Wei, Z., and Conlon, E. M. (2015). Parallel Markov chain Monte Carlo for non-Gaussian posterior distributions. Stat, 4:304-319.

Oldenborgh, G. J. v. (2018). Extreme rainfall in japan, 2018 - a quick look. 
Table 9. 95\% CIs of common rainfall mean in five Thailand's regions.

\begin{tabular}{lrrr}
\hline \multirow{2}{*}{ Methods } & \multicolumn{2}{c}{$95 \%$ CIs for $\vartheta$} & \multirow{2}{*}{ Lengths } \\
\cline { 2 - 3 } & \multicolumn{1}{c}{ Lower } & \multicolumn{1}{c}{ Upper } & \\
\cline { 1 - 3 } On August 5,2020 & & \\
FGCI & 2.5545 & 6.3342 & 3.7798 \\
LS & 3.2166 & 5.6846 & 2.4681 \\
MOVER & 2.7216 & 9.0296 & 6.3080 \\
PB & 5.8876 & 11.4965 & 5.6089 \\
HPD-JR & 3.5216 & 7.8533 & 4.3317 \\
HPD-NGB & 2.4969 & 6.0904 & 3.5935 \\
\hline On August & 9,2020 & & \\
FGCI & 7.1127 & 16.8809 & 9.7682 \\
LS & 10.4880 & 16.0363 & 5.5483 \\
MOVER & 7.5814 & 23.3171 & 15.7357 \\
PB & 14.5229 & 23.5821 & 9.0591 \\
HPD-JR & 12.8404 & 20.4349 & 7.5945 \\
HPD-NGB & 7.2928 & 17.1265 & 9.8337 \\
\hline
\end{tabular}

Oldenborgh, G. J. v., Wiel, K. v. d., Philip, S., Kew, S., Antonia, S., Otto, F., Haustein, K., Roop, S., Arrighi, J., and Vecchi, G. (2019). Rapid attribution of the extreme rainfall in texas from tropical storm imelda.

Otto, F. and Oldenborgh, G. J. v. (2017). UK storm desmond revisited, december 2017.

Owen, W. J. and DeRouen, T. A. (1980). Estimation of the mean for lognormal data containing zeroes and left- censored values, with applications to the measurement of worker exposure to air contaminants. Biometrics, 36(4):707-719.

Shimizu, K. and Iwase, K. (1981). Uniformly minimum variance unbiased estimation in lognormal and related distributions. Communications in Statistics - Theory and Methods, 10(11):1127-1147.

Supasod, W. (2006). Development of Water Resources for Agriculture. Technical report. Available from: http://www.rid.go.th/thaicid/_5_article/2549/03Water_Agri.pdf.

Tian, L. (2005). Inferences on the mean of zero-inflated lognormal data: the generalized variable approach. Statistics in Medicine, 24(20):3223-3232.

Tian, L. and Wu, J. (2007). Inferences on the Common Mean of Several Log-Normal Populations: The Generalized Variable Approach. Biometrical Journal, 49(6):944-951.

Tierney, W. M., Overhage, J. M., Murray, M. D., Harris, L. E., Zhou, X.-H., Eckert, G. J., Smith, F. E., Nienaber, N., McDonald, C. J., and Wolinsky, F. D. (2003). Effects of computerized guidelines for managing heart disease in primary care. Journal of General Internal Medicine, 18(12):967-976.

Wu, W.-H. and Hsieh, H.-N. (2014). Generalized confidence interval estimation for the mean of deltalognormal distribution: an application to New Zealand trawl survey data. Journal of Applied Statistics, 41(7):1471-1485.

Zhou, X.-H., Gao, S., and Hui, S. L. (1997). Methods for Comparing the Means of Two Independent Log-Normal Samples. Biometrics, 53(3):1129-1135.

Zhou, X. H. and Tu, W. (2000). Confidence intervals for the mean of diagnostic test charge data containing zeros. Biometrics, 56(4):1118-1125.

Zou, G. Y., Taleban, J., and Huo, C. Y. (2009). Confidence interval estimation for lognormal data with application to health economics. Computational Statistics \& Data Analysis, 53(11):3755-3764. 


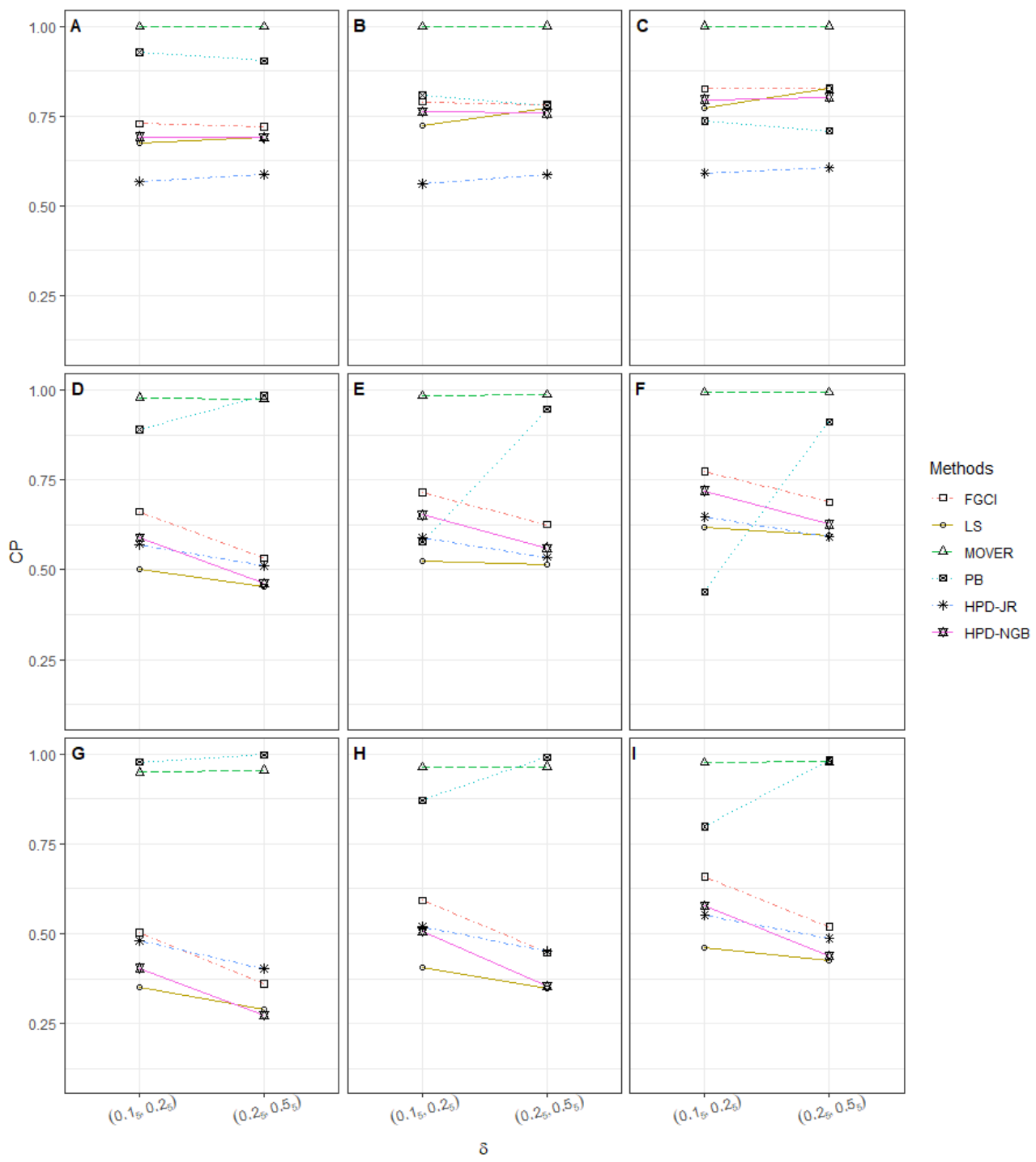

Figure 3. CP performances of $95 \% \mathrm{CIs}$ for $\vartheta$ : 10 sample cases in the following cases (sample sizes, variances): (A) $\left(30_{5}, 50_{5}, 1_{2}, 2_{5}\right)$, (B) $\left(30_{5}, 50_{5}, 2_{5}, 4_{5}\right)$, (C) $\left(30_{5}, 50_{5}, 3_{5}, 5_{5}\right)$, (D) $\left(30_{3}, 50_{3}, 100_{4}, 1_{2}, 2_{5}\right)$, (E) $\left(30_{3}, 50_{3}, 100_{4}, 2_{5}, 4_{5}\right)$, (F) $\left(30_{3}, 50_{3}, 100_{4}, 3_{5}, 5_{5}\right)$, (G) $\left(50_{5}, 100_{5}, 1_{5}, 2_{5}\right)$, (H) $\left(50_{5}, 100_{5}, 2_{5}, 4_{5}\right)$, (I) $\left(50_{5}, 100_{5}, 3_{2}, 5_{3}\right)$. 
A

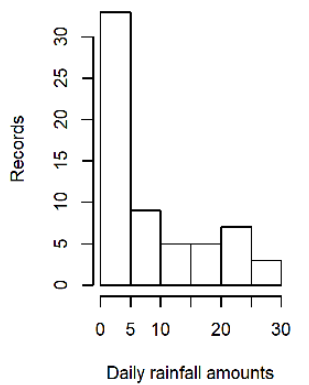

B

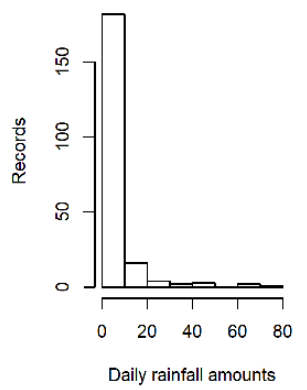

C

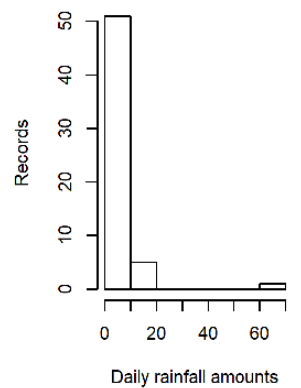

E
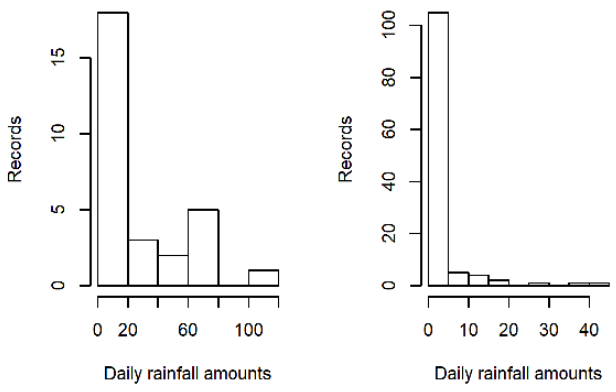

Figure 4. Histogram plots of daily rainfall data in five Thailand's regions on August 5, 2019: (A) Northern (B) Northeastern (C) Central (D) Eastern (E) Southern.

A

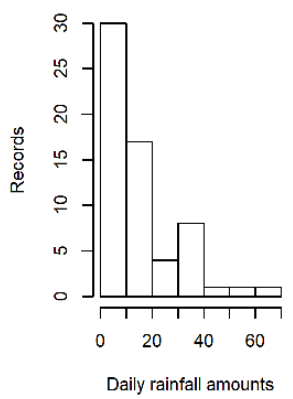

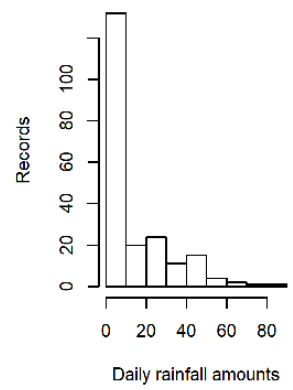

$c$

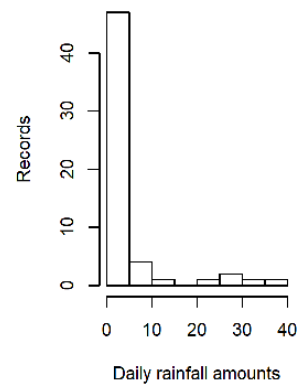

E
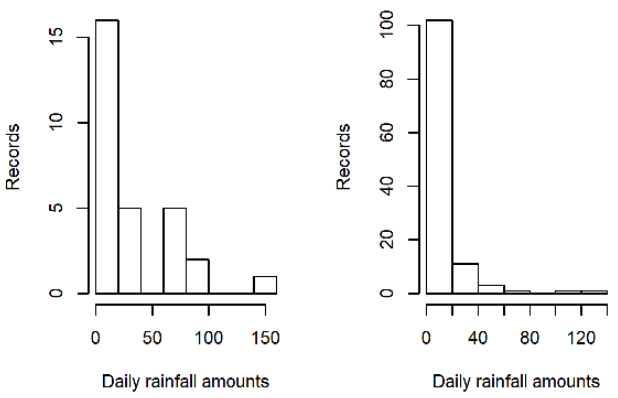

Figure 5. Histogram plots of daily rainfall data in five Thailand's regions on August 9, 2019: (A) Northern (B) Northeastern (C) Central (D) Eastern (E) Southern. 

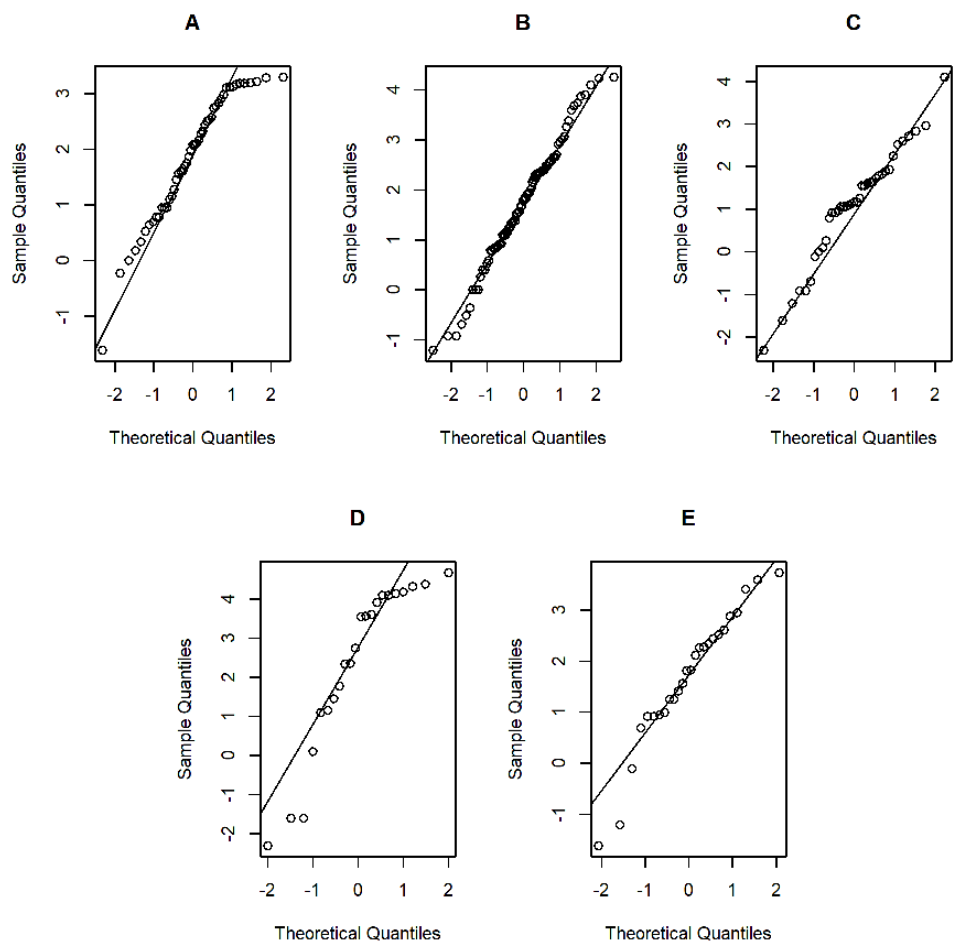

Figure 6. Normal Q-Q plots of log-positive daily rainfall data in five Thailand's regions on August 5, 2019: (A) Northern (B) Northeastern (C) Central (D) Eastern (E) Southern.
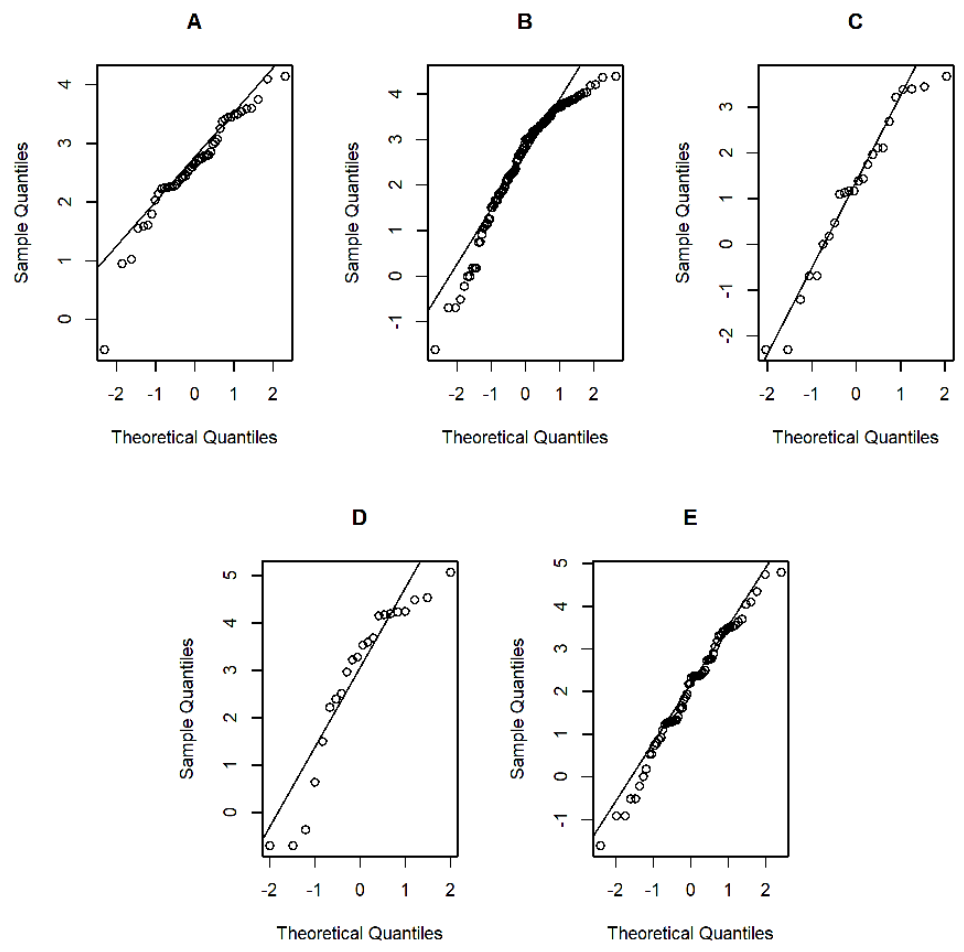

Figure 7. Normal Q-Q plots of log-positive daily rainfall data in five Thailand's regions on August 9, 2019: (A) Northern (B) Northeastern (C) Central (D) Eastern (E) Southern. 